II.

\title{
Scientia, Dolus und Error bei der Stellvertretung nach klassischem römischen Recht.
}

\author{
Von \\ Herrn Professor Dr. Fritz Schulz \\ in Kiel.
}

Es ist ein altes, viel behandeltes Problem, das im folgenden noch einmal für das römische Recht der Klassiker untersucht werden soll: wenn die Wirksamkeit eines Rechtsgeschäfts bestimmte psychologische Tatbestände zur Voraussetzung hat, wessen Psyche ist dann bei einem von einem Stellvertreter geschlossenen Geschäfte maßgebend, die des Vertreters oder die des Vertretenen? Das deutsche BGB. beantwortet diese Frage im $\S 166$ :

nSoweit die rechtlichen Folgen einer Willenserklärung durch Willensmängel oder durch die Kenntnis oder das Kennenmüssen gewisser Umstände beeinflußt werder, kommt nicht die Person des Vertretenen, sondern die des Vertreters in Betracht.

Hat im Falle einer durch Rechtsgeschäft erteilten Vertretungsmacht (Vollmacht) der Vertreter nach bestimmten Weisungen des Vollmachtgebers gehandelt, so kann sich dieser in Ansehung solcher Umstände, die er selbst kannte, nicht auf die Unkenntnis des Vertreters berufen. Dasselbe gilt von Umständen, die der Vollmachtgeber kennen mußte, sofern das Kennenmüssen der Kenntnis gleichsteht."

Mit dieser Vorschrift übernimmt das Gesetzbuch nur, was Windscheid seinerzeit als gemeines römisches Recht gelehrt hatte; nach Windscheids Auffassung berücksichtigte auch das römische Recht grundsätzlich allein die Person des Vertreters, die Unkenntnis des Vertreters aber kam dem Vertretenen nicht zustatten, wenn in einem Punkte, in welchem der Vertreter konform der Instruktion des Vertretenen han- 
delte, dieser die Sachlage kannte. ${ }^{1}$ ) Freilich mußte aber auch Windscheid anerkennen, daß „die Quellen kein reines Resultat gewährten"..2) In der Tat sind die Widersprüche unter den Quellenentscheidungen nicht wegzuleugnen, und vergebens haben die Romanisten von der Glosse bis zum BGB. mit allen Hebeln und Schrauben der Panharmonistik versucht, die disparaten Fragmente ins Lot zu bringen. ${ }^{3}$ )

Im folgenden soll die alte Pandektenkontroverse noch einmal mit den modernen Forschungsmitteln aufgenommen werden. Die einschlägigen Quellenstellen sind bisher - soviel ich sehe - mit der interpolationenkritischen Methode gründlich noch nicht untersucht worden: A. Pernice hat unser Problem nur in seinem ersten, noch vor der Periode der Interpolationenforschung liegenden Labeo-Bande flüchtig gestreift ${ }^{4}$ ); Mitteis ist in seinen Ausführungen über die römische Stellvertretung im ersten Bande seines Römischen Privatrechts auf unsere Fragen nicht zu sprechen gekommen, seine Monographie über die Stellvertretung aber (1885) liegt noch vor dem Aufkommen der neuen Richtung. Vor allem

1) So die Formulierung der Windscheidschen Lehre durch Kipp bei Windscheid Pand. $1 \$ 73$ N. 18. Windscheid selbst hat seine Auffassung nicht recht geschickt zum Ausdruck gebracht. $-{ }^{2}$ ) A.a. O. N. 21 am Ende. - s) Aus der gemeinrechtlichen Literatur sind folgende Arbeiten hervorzuheben: Das Quellenmaterial hat die Glosse bereits im wesentlichen vollständig zusammengetragen, auch die vielen Widersprüche unter den Entscheidungen richtig erkannt, natürlich auch in ihrer Art "gelöst". Sedes materiae ist für die Glosse 1.51 D.21, 1, zu welcher Stelle die Glosse eine ausführliche Erörterung aus der CodexSumme des Azo (aus dem Titel de aediliciis actionibus) wörtlich rezipiert hat. - Cuiacius ad Afric. Tract. VIII (zu der zitierten 1.51) Op. 1,1306 ff. (Venet. 1758). Eine sehr ausführliche Erörterung der 1.51 cit. gibt Bartholomaeus $\mathrm{C}$ hesius Interpretationes Juris II cap. 25 (Heineccius, Jurisprudentia Romana et Attica II, $475 \mathrm{ff}$.). - Aus der Literatur des 19. Jahrhunderts: Glück 20, 51 (1819); Mühlenbruch, Die Lehre von der Cession der Forderungsrechte (3. Aufl. 1836) $\$ 13$ S. 117 ff.; Herrmann, Zeitschr. f. ZivilR. und Prozes N. F. 7,269 (1850); Buchka, Die Lehre von der Stellvertretung $S .13$ f. und S. 58 f. (1852); Alb. Schmid, Grundlehren der Cession Bd. 2 pass. (1866); Mandry, D. gemeine Familiengüterrecht Bd. 1 (1871) S. 120 f., 127 f.; Curtius, ArchZivPrax. 58, 88 ff. (1875); Hellmann, Stellvertretung S. 152 f., $155 \mathrm{ff}$. (1882); Mitteis, Stellvertretung $\$ \S 33,35,36$ (1885); Dunkhase, ArchZivPrax, 77, 119 ff. (1891). - 4) S. 502 f. 
dem modernen Rechte gewidmete Arbeiten, wie der zweite Band von Leonhards Irrtumslehre ${ }^{1}$ ) und Hupkas Vollmacht ${ }^{2}$ ) behandeln zwar auch einen Teil der hier zu untersuchenden römischen Quellenstellen, verwenden aber gleichfalls die interpolationenkritische Methode nicht. Unter Verwendung dieser Methode hat freilich jüngstens Solazzi in seinem Aufsatze Errore e Rappresentenza ${ }^{3}$ ) unser Problem behandelt; seine Quellenanalyse ist jedoch nicht gründlich genug, und so hat er von den zahlreichen hier vorliegenden Interpolationen nur zwei erkannt.

M. E. läßt sich klipp und klar erweisen, daß die Widersprüche, an denen sich die gemeinrechtlichen Dogmatiker abgemüht baben, den Kompilatoren zu verdanken sind; schwieriger ist es natürlich - hier wie überall -, die Grundsätze des klassischen Rechts zu erkennen und zu sagen, wohin die Kompilatoren mit ihren Eingriffen hinauswollten.

Die Lehre der Klassiker ist m. E. die gewesen. Geschieden wird zunächst zwischen indirekter und direkter Vertretung.

Bei der indirekten Vertretung, wie sie regelmäßig bei der Vertretung durch gewaltfreie Personen gegeben ist, wird ausschließlich die Person des Vertreters berücksichtigt. Für die scientia formuliert heißt das: im Rechtsverhältnis zwischen Vertreter und Drittkontrahenten entscheidet ausschließlich die Kenntnis des Vertreters. Kenntnis und Unkenntnis des Prinzipals ist unbeachtlich.

1) Der Irrtum als Ursache nichtiger Verträge 2, $115 \mathrm{ff.} \mathrm{(2.} \mathrm{Aufl.}$ 1907; Studien zur Erläuterung des bürgerlichen Rechts, Heft 23). Der Versuch Leo nhards, die einzelnen hierher gehörigen Entscheidungen $\mathrm{zu}$ isolieren und jede für sich aus den Besonderheiten des Falls zu erklären, scheint mir nicht gelungen; meine Untersuchung ergibt umgekehrt, dafi die Klassiker die Fälle durchaus nach einheitlichen Gesichtspunkten entschieden haben. $-{ }^{2}$ ) S. $63 \mathrm{ff}$. Nur die Interpolation von fr. 17 D. 40,12 hat $H$ upka im ganzen richtig erkannt (S. 65 N.2), wenn auch nicht bewiesen; vgl. unten S. $61 .{ }^{3}$ ) Rivista italiana per le scienze giuridiche 50 (1911) Fasc. II--III (zitiert nach dem Sonderdruck). Diese Arbeit wurde mir erst nach Absendung des Manuskripts an die Redaktion bekannt, ist aber im folgenden vollständig berücksichtigt. 
Bei der direkten Vertretung, wie sie regelmäßig nur bei den Geschäften der Gewaltunterworfenen möglich ist, unterscheiden die Klassiker weiter, und $\mathrm{zwar}$ weisen unsere Quellen mehrere derartiger Unterscheidungen auf: man will die Person des Gewaltunterworfenen berücksichtigen, wenn diesem beim Abschluß des Geschäftes eine gewisse Selbständigkeit eingeräumt worden ist, über das dazu erforderliche Maß dieser Selbständigkeit aber ist man sich nicht einig gewesen.

1. Als Hauptunterscheidung tritt in unsern Quellen auf die Unterscheidung von Geschäften peculiari nomine und domini bez. patris nomine. "Im Namen" des peculium oder des Herrn hat für die Klassiker nicht die Bedeutung, die wir heute mit dieser Formel zu verbinden gewöhnt sind. ${ }^{1}$ ) Entscheidend ist die wirtschaftliche Beziehung des Geschäfts zum Peculiar- oder Herrenvermögen; geht das Geschäft das peculium wirtschaftlich nichts an, dient es nicht der Verwaltung und Bewirtschaftung des peculium, so ist es nicht peculiari nomine geschlossen. Die Tragweite dieser Unterscheidung ist nun die:

a) Ist das Geschäft peculiari nomine vorgenommen, so ist grundsätzlich, wie bei der indirekten Vertretung, allein die Person des Vertreters (des Gewaltunterworfenen) maßgebend. Soweit also die Kenntnis eines Umstandes schädlich ist $^{2}$, heißt das: Kenntnis des Vertreters schadet dem Geschäftsherrn ungeachtet seiner Unkenntnis; Unkenntnis des Vertreters nützt dem Geschäftsherrn ungeachtet seiner eigenen Kenntnis. Doch bedarf der zweite Teil dieser These alsbald eines Zusatzes: es gibt eine Reihe von Entscheidungen ${ }^{3}$ ), die ich für echt halte, und die die Kenntnis des Geschäftsherrn trotz Unkenntnis des Vertreters für schädlich erklären, bald schlechthin, bald mit Einschränkungen; eine herrschende Lehre ist nicht zu erkennen.

b) Ist das Geschäft domini vel patris nomine vorgenommen, so ist umgekehrt ausschließlich die Person des

1) Vgl. dazu Victor Bruns, Besitzerwerb durch Interessevertreter S. 28 und 31 N. 2, 3; Rosenberg, Stellvertretung im Prozek S. 356. - 2) Mitunter nutzt sie, wie beim Erbschaftserwerb; vgl. unten S.66.

3) Unten S. 55 f. 63 . 
Vertretenen maßgebend: Kenntnis des Vertreters schadet also nicht, Unkenntnis des Vertreters nützt nicht, wenn der Geschäftsherr Kenntnis hatte.

2. Neben dieser Hauptunterscheidung findet sich aber in den Quellen eine zweite: die Unterscheidung von Geschäften mandatu domini vel patris und Geschäften sine mandatu. Die juristische Tragweite dieser Unterscheidung ist die gleiche wie die der ersten, so zwar, daß das mandierte Geschäft behandelt wirả, wie ein Geschäft domini nomine, das nicht mandierte wie ein Peculiargeschäft. Es ist ohne weiteres klar, daß sich die beiden Distinktionen nicht decken; die Peculiargeschäfte sind zwar ohne Auftrag geschlossen, aber nicht jedes auftraglose Geschäft ist ein Peculiargeschäft.

3. Von einer dritten Unterscheidung ist nur eine schwache Spur erkennbar: die Geschäfte, die der Gewaltunterworfene certum mandante patre vel domino geschlossen hat, werden allen übrigen gegenübergestellt; die Bedeutung dieser Unterscheidung ist die, daß das Geschäft certum patre mandante behandelt wird wie das Geschäft domini nomine unter 1, alle übrigen wie die Geschäfte peculiari nomine.

Das Gemeinsame aller dieser drei Unterscheidungen liegt, wie gesagt, darin, daß überall der Seelenzustand des Vertreters maßgebend sein soll, wenn er bei der Vornahme des Geschäftes einigermaßen selbständig gehandelt hat: dabei legt man bald Gewicht auf die wirtschaftliche Selbständigkeit des Peculiargeschäfts, bald auf die Initiative des Vertreters (sine mandatu), bald auf die Beteiligung des Vertreters bei der Bildung des Geschäftswillens (sine certo mandatu). -

Die Kompilat ore n beabsichtigten, diese klassische, in der Tat ja auch zum Teil unfertig gebliebene, Lehre wesentlich $\mathrm{zu}$ vereinfachen und $\mathrm{zwar}$ in folgender Weise: $\mathrm{ma}$ agebend soll sein sowohl die Person des Vertreters wie die des Vertretenen; ist also die Kenntnis eines Umstandes schädlich, so schadet sowohl die Kenntnis des Vertreters wie die des Vertretenen und zwar bei der direkten Vertretung so gut wie bei der indirekten. Die Veränderungen, die die Kompilatoren zu diesem $\mathrm{Z}_{\text {wecke }}$ an den klassischen Texten vorgenommen haben, sollen durch die folgende Quellenanalyse erwiesen werden. 
1. Kenntnis der Verbotswidrigkeit eines Geschäfts (Kauf einer res litigiosa, Geschäfte in fraudem creditoru m).

$$
\text { D. }(44,6) 2 \text { : }
$$

Ulpianus libro sexto fideicommissorum.

Si servus cum emerit scit, ignoravit autem dominus, vel contra, videndum est, cuius potius spectanda sit scientia. et magis est, ut scientia inspicienda sit eius qui comparavit, non eius, cui adquiretur, et ideo poena litigiosi competit ${ }^{1}$ ), sic tamen, si non mandatu domini emit: nam si mandatu, etiamsi scit servus, dominus autem ignoravit, scientia non nocet: et ita Iulianus in re litigiosa scribit.

Die Interpolation der Stelle hat bereits Lenel erkannt: "Ulpianum enim hoc loco non de litigiosis egisse apparet ex extremis huius fr. verbis ". ${ }^{2}$ ) In der Tat, wenn Ulpian sagt: so entscheidet Julian beim Kauf einer res litigiosa, so muß er selbst (Ulpian) offenbar von einem andern, wenngleich ähnlichen Fall reden, sonst hätte ein einfaches „et ita Iulianus scribit" genügt. Andre Interpolationsindizien außer dem von Lenel angegebenen kommen hinzu. "Poena competit" kommt in den Pandekten außer an dieser Stelle nur noch einmal vor $\left.^{3}\right)$, in D. $(49,16), 3,9$, wo der Ausdruck gleichfalls unecht ist ${ }^{4}$ ); bei Justinian dagegen findet sich z. B. gerade in der von der res litigiosa handelnden Novelle 112 C. 2 \$ "poena videlicet ex bac lege competente“. Das Wort "ideo" hat keinen Sinn, mit demselben Rechte könnte es nideo poena non competit" heißen, denn im Vorangehenden sind zwei Fälle aufgeführt (1. si servus scit, ignoravit autem dominus, 2. vel contra, also: si dominus scit, servus ignoravit) und nur für den ersten Fall ist die Entscheidung „et ideo poena competit" zutreffend. Schließlich sagt der Satz auch sachlich unzutreffendes: die Geldstrafe des Augusteischen

1) Bei der Wiedergabe der Quellentexte wird folgende Technik verwandt: Zusätze der Kompilatoren sind kursiv, gestrichene klassische Stücke petit gedruckt. - ${ }^{2}$ ) Palingenesia Ulpian Nr. 1902. - ${ }^{3}$ ) Voc. Jur. Rom. 1, 847 Z. 42. - ) Gradenwitz, Savignyzeitschrift 7, 58 ff.; Krüger, in seiner Digestenausgabe zu der Stelle; Lenel, Paling. Modestin Nr. 167 N.1. 
Edikts $\left.{ }^{1}\right)$ kann bei der Vermögensunfähigkeit des Sklaven natürlich niemals diesen ${ }^{2}$, sondern immer nur den Herrn treffen, diesen aber selbstredend nur, wenn er die Streitbefangenheit der Sache kannte. Nach unserer Stelle aber soll trotz Unkenntnis des Herrn bei Kenntnis des Sklaven die Strafe verfallen.

Nicht also von der Strafe des Augusteischen Edikts handelte Julian, sondern von der Einrede, die der Vindication des Käufers einer streitbefangenen Sache entgegengehalten werden konnte: si fundum litigiosum sciens a non possidente emeris eumque a possidente petas, opponitur tibi exceptio, per quam omni modo summoveris. ${ }^{3}$ j Damit die exceptio durchgreift, ist scientia des Erwerbers erforderlieh; ist nun dieser ein Gewaltunterworfener, so entsteht die Frage, ob es auf seine Kenntnis oder auf die des Gewalthabers ankommt. Julian beantwortet diese Frage folgendermaßen: ist das Geschäft nicht im Auftrage des Gewalthabers vorgenommen, so entscheidet allein die Kenntnis des Gewaltunterworfenen; Kenntnis des Sklaven schadet also trotz Unkenntnis des Herrn, Kenntnis des Herrn bei Unkenntnis des Sklaven (dieser Fall wird ausdrücklich ("vel contra"!) mit aufgeführt und mit entschieden) ist unschädlich. Hatte der Herr zu dem Geschäfte Auftrag gegeben, so schadet die Kenntnis des Sklaven bei Unkenntnis des Herrn nichts; daß auch umgekehrt die Kenntnis des Herrn in diesem Falle trotz Unkenntnis des Sklaven schadet, ist zwar in dieser Stelle nicht ausgesprochen, gleichwohl aber Julians Meinung, wie sich aus dem unten zu erörternden fr. 51 D. 211 ergibt. ${ }^{4}$ ) Die gemeinrechtlichen Interpreten haben $z$ war vielfach behauptet, Julian meine bei den Worten "nam si mandatu" nur das Spezialmandat ${ }^{5}$ ), doch diese Beschränkung ist weder

1) Fr. de iure fieci $\left.\$ 8 .-{ }^{2}\right)$ Man hat gemeint. die Geldstrafe treffe das peculium des Sklaven; so Mühlenbruch 128 N.258; Dunkhase 128. Selbst wenn der Gedanke klassisch wäre, so fehlt in unserm Text doch selbst ein Hinweis darauf, dak der Sklave im gegebenen Falle uberhaupt ein peculium hat. - ${ }^{3}$ ) Gaius 4,117. - 4) Unten S. 45. s) So schon die Glosse si mandato: hic de certo emendo mandavit, puta Sticho, quo casu ipse dominus videtur emisse. Cuiacius ad h. l. (Op.1,1308); Pernice, Labeo 1, 503; Hellmann 161; Mitteis 281; Dunkhase 128. 
aus dem Tatbestand noch aus der Entscheidung ersichtlich und daher abzulehnen.

Julians Entscheidung ist also vollkommen klar; auf welchen Fall wollte sie aber wohl Ulpian analog anwenden? Wahrscheinlich das Richtige trifft Lenels Vermutung ${ }^{1}$ ), Ulpian habe von einer Veräußerung in fraudem missionis fideicommissorum servandorum causa gesprochen; hier mußten ja natürlich analoge Fälle auftauchen. Hervorzuheben ist freilich, daß Ulpian sich bei der Veräußerung in fraudem creditorum nicht unserm fr. 2 entsprechend entscheidet:

$$
\text { D. }(42,8) 6,12
$$

Ulpianus libro sexagesimo sexto ad edictum. Simili modo quaeritur, si servus ab eo, qui solvendo non sit, ignorante domino ipse sciens rem acceperit, an dominus teneretur. et ait Labeo hactenus eum teneri, ut restituat quod ad se pervenit aut dumtaxat de peculio damnetur vel si quid in rem eius versum est. eadem in filio familias probanda sunt. ${ }^{2}$ ) sed si dominus scit, suo nomine convenietur.

Ulpian unterscheidet hier nicht zwischen beauftragter und unbeauftragter Geschäftsführung, aber selbst wenn man die Entscheidung ausschließlich auf ein Geschäft ohne Auftrag des Herrn bezieht, so weicht sie doch noch von der unsres fr. $2 \mathrm{ab}$ : in fr. 2 wird ausdrücklich bei auftragsloser Geschäftsführung die Kenntnis des Herrn für unschädlich erklärt, hier aber soll sie schaden. Eine Interpolation des Schlußsatzes des fr. $6 \S 2$ ist damit aber noch keineswegs erwiesen, denn der gleiche Gedanke taucht auch noch in anderen sicher echten Texten auf. Ein Widerspruch Ulpians mit sich selbst in zwei verschiedenen Werken hat durchaus nichts Unwahrscheinliches. -

2. Kenntnis der Mangelhaftigkeit der Kaufsache.

$$
\text { D. }(21,1) 51 \text { : }
$$

Africanus libro octavo quaestionum.

Cum mancipium morbosum vel vitiosum servus emat et redhibitoria vel ex empto ${ }^{3}$ ) dominus experiatur, omnimodo

1) A. a. O. $-{ }^{2}$ ) Diese Worte sind wohl kompilatorische Auffüllung, denn im nächsten Satz ist wieder allein vom dominus die Rede. Die gleiche Interpolation unten S. 70. - 3) Möglich, dak die Worte 
scientiam servi, non domini spectandam esse ait, ut nihil intersit, peculiari an domini nomine emerit et certum incertumve mandante eo emerit, quia tunc et illud ex bona fide est servum, cum quo negotium sit gestum, deceptum non esse, et rursus delictum eiusdem, quod in contrahendo admiserit, domino nocere debet. sed si servus mandatu domini hominem emerit, quem dominus vitiosum esse sciret, non tenetur venditor.

1. Circa procuratoris personam, cum quidem ipse scierit morbosum vitiosum esse, non dubitandum, quin, quamvis ipse domino mandati vel negotiorum gestorum actione sit obstrictus, nihilo magis eo nomine agere possit: at cum ipse ignorans esse vitiosum mandatu domini qui id sciret emerit et redhibitoria agat, ex persona domini utilem exceptionem ei non putabat opponendam.

Wie das "ait" und "putabat" zeigt, ist auch hier wieder Julian der Entscheidende. ${ }^{1}$ ) Die Stelle ist seit Alters eine crux für die Ausleger, wie so häufig deshalb, weil sie schwer interpoliert ist.

Das Pr. steht zunächst sachlich im Widerspruch mit dem soeben erörterten fr. 2, sodann aber auch mit sich selbst.

Es steht im Widerspruch mit fr. 2: denn nach diesem schadet die Kenntnis des Sklaven dem Herrn bei beauftragter Geschäftsführung nicht, in unserm pr. aber wird ausdrücklich das Gegenteil behauptet. Man hat diesen Widerspruch hinwegdeuten wollen, aber ohne Erfolg. Die Glosse ${ }^{2}$ ) faßte im Anschluß an $\mathrm{Azo}^{3}$ ) in fr. 2 das Mandat als Spezialauftrag

vel ex empto Interpolation oder Glossem sind (Solazzi 10 N. 1); darauf kommt für uns nichts an.

1) Pernice, Labeo 1,503 N. 34. - 2) Gl. Cum mancipiun. 3) Azo, Summa Codicis im Titel de aediliciis actionibus (Exempl. von 1482 Petr. Drach Spir.): Si autem quaeras, au servi ementis scientia vel alterius qui emit nomine meo mihi nocere debeat, distingue in servo, nomine peculiari an nomine meo. Si nomine peculiari, sive cum mandato sive sine mandato, scientia et ignorantia servi tantum et non mea inspicitur ut D. eod. l. cum mancipium (unsere lex) et D. de lib. causa 1 . immo $\S$ tunc $(40,12,16,2)$ et $D$. de litig. 1.2 primum responsum. Excipitur quando me praesente et non contra dicente servus emit, nam mea scientia in meum praeiudicium inspicitur ut D. de lib. causa 1 . immo $\S$ plane si filius $(40,12,16,4)$. Si vero praesens contradicere non possem, non obesset mihi mea scientia ut D. de contr. 
und bezog in fr. 51 die Worte "certum incertumve mandante" auf das Kontrahieren peculiari nomine.

Gl. Certum: Repete: et cum peculiari nomine emit, non interesse, certum incertumve emerit eo mandante.

Sie gelangte damit zu folgendem System: 1. Kontrahieren peculiari nomine: allein die Person des Vertreters ist maßgebend, mag Auftrag vorliegen oder nicht; 2. Kontrahieren nomine domini ohne Spezialauftrag: es entscheidet gleichfalls die Person des Vertreters; 3. Kontrahieren nomine domini mit Spezialauftrag: maßgebend ist umgekehrt ausschließlich die Person des Gewalthabers. Nicht minder gewaltsam war aus der neueren Literatur die Vereinigung Hellmanns ${ }^{1}$ ): auch er nahm in Fr. 2 einen Spezialauftrag an, die Worte "certum incertumve mandante" unsres Pr. sollten dagegen bedeuten: Vollmacht zum Kauf einer bestimmten Gattung (certum) oder Kaufvollmacht im Allgemeinen (incertum). Aber weder ist es gestattet, Fr. 2 auf den Spezialauftrag zu. beschränken, noch darf man African zutrauen, daß er den Ausdruck certum mandare ohne weiteren Zusatz zur Bezeichnung des Gattungsmandats verwendet haben sollte, wo ihm doch klar sein mußte, daß man darunter gerade so gut das Spezialmandat verstehen, mindestens mitverstehen konnte. ${ }^{2}$ )

empt. 1. in huiusmodi $(18,1,12)$. Si servus autem emerit nomine meo et mandato meo, non autem peculiari nomine, si quid mandavi ei de incerto idem est quod supra. Idemque est etiam si sine mandato meo quis mihi emerit servum vitiosum ut D. eod. 1 . cum manipium $\$$ circa (unsere lex $\$ 1$ ). Si vero mandavero servo meo vel alii de certo servo emendo, mea scientia et ignorantia tantum inspicitur ut $D$. de lib. causa 1 . immo $\S$ tunc $(40,12,16,2)$ et 1 . in servo $(40,12,17)$ et $\mathrm{D}$. de litigios. 1 : 2 secund. respons. $(44,6,2 \mathrm{Satz} 2)$ et D. de contr. empt. l. sed et si servo $(18,1,13)$ et D. eod. l. cum mancipium $\S$ sed si servus (unsere lex pr. letzter Satz). - Nec obstat quod ibidem dicitur "certum incertumve", quia refertur ad eam causam, quando emit nomine peculiari. In usucapione quoque idem distinguendum est ut probatur D. pro emptore l. 2 \$ si servus usque ad illum „si a pupillo" $(41,4,2,10-14)$. - Illud certum est, quod, ubi quis emit nt nuncius, nunquam eius scientia vel ignorantia spectatur sed domini.

1) S. 159. $\left.-{ }^{2}\right)$ Gegen Hellmann auch Mitteis 285 N. 375 und jetzt Solazzi 4 N. 1. 
Willkürlich war es schließlich auch, in Fr. 2 in dem Mandatar einen bloßen Boten zu sehen und so den Widerspruch beheben zu wollen. ${ }^{1}$ )

Die Meisten haben denn auch diesen Widerspruch zwischen Fr. 51 pr. und Fr. 2 zugegeben und waren nur bestrebt, ihn zu erklären und zu rechtfertigen. Weit verbreitet war die Meinung, Ulpian erkläre in Fr. 2 deshalb die Kenntnis des Sklaven bei beauftragter Geschäftsführung für unschädlich, weil "die Verwirkung der poena litigiosi in Frage stehe; in allen Straffällen aber, bei welchen es sich nicht geradezu um etwas Verbrecherisches handele, werde dem auf Befehl des Gewalthabers handelnden Sklaven nachgesehen; der Gewalthaber selbst aber könne die Poen natürlich nur dann verwirken, wenn in seiner Person die Voraussetzungen derselben vorhanden waren ". ${ }^{2}$ ) Dieser Erklärungsversuch bricht jetzt ohne Weiteres zusammen, nachdem oben dargelegt worden ist, daß Fr. 2 in seiner klassischen Form gar nicht von der poena litigiosi redet. Eine dogmatisch interessante, historisch freilich gleichfalls unzulängliche Erklärung gab Bachov: im Fr. 51 handele es sich um die Wirkung der Kenntnis gegenüber dem Mitkontrahenten (dem Verkäufer), dessen Vertrauen (auf die Kenntnis des Käufers) müsse geschützt werden; in Fr. 2 dagegen stehe nicht das Verhältnis gegenüber dem Verkäufer, sondern gegenüber einem am Vertrage nicht Beteiligten in Frage (dem beklagten Besitzer der Streitsache), hier sei mithin ein Vertrauensschutz nicht erforderlich. ${ }^{3}$ ) Bekanntlich ist diese Erklärung - unabhängig

1) So Jhering in seinem Jahrb. 1, 290; Curtius 92; dagegen Dunkhase' 128. - ${ }^{2}$ ) So Cuiacius ad Afr. ad h. 1. Op. 1, 1308; Mühlenbruch 128 N. 258; Buchka 15 N. 17; Dunkhasé 129; R. Leonhard 128. - 3) Notae et Animadversiones ad vol. posterius disputationum H. Treutleri Tom. 2, Pars 1 Disp. 2, Thes. 3 sub C S. 72 : Quod in 1.51 in pr. dicitur omnimodo scientiam servi nocere domino, etiamsi mandante domino emerit, ei obstare videtur 1.2 de litigios., nisi quis fortasse dixerit, oppositionem istam, quod scienta servi noceat, non ubique neque in omnibus, sed hoc nostro casu obtinere: quo causa venditoris, qui bona fide cum servo contraxit et probabilem causam habuit existimandi, dominum vitium illud non latuisse, causa domini potior est. Quod si dominus non cum venditore, sed cum alio concurrat, aut de poena quadam agatur, in eiusmodi casibus ignorantia domini proderit ad excusationem. 
von Bachov - von Mitteis seinerzeit wieder aufs Neue vertreten worden ${ }^{1}$ ), doch ist den Klassikern wohl die Idee, bei der Stellvertretung dem Drittkontrahenten einen Vertrauensschutz zu gewähren, vollständig fremd. ${ }^{2}$ )

Das Pr. des Fr. 51 widerspricht sich aber außerdem noch selbst. ${ }^{3}$ ) Im Eingang heißt es, auch bei der beauftragten Vertretung ist, mag bestimmtes oder unbestimmtes Mandat vorliegen, nie auf die Kenntnis des Herrn zu sehen: scientiam servi non domini (scil. scientiam) spectandam esse, denn zu "domini" ist natürlich "scientiam" hinzuzudenken. Am Schlusse des Pr. aber wird gerade das Gegenteil gesagt: bei beauftragter Vertretung kommt doch die Kenntnis des Herrn in Betracht. Schon Rogerius hat dies erkannt:

Gl. Sed si servus: Rogerius dixit, quod iste versus nsed $\mathbf{s i}^{\text {" corrigit praedicta. }}$

und die Glosse glaubte auf folgende gewaltsame Weise helfen zu müssen.

Gl. non domini: scilicet ignorantiam.

In der Tai wenn man den Eingang liest: scientiam servi, non domini ignorantiam spectandam esse, so ist der Widerspruch mit dem Schlußsatze geschwunden, nur daß diese Ergänzung sprachlich ganz unmöglich ist. Etwas geschickter halfen die Späteren. Im ganzen ersten Teil des Pr., erklärte man, wird vorausgesetzt, daB mindestens der Sklave in Kenntnis war: „auf die Kenntnis des Herrn ist nicht zu sehen", nämlich dann, wenn der Vertreter Kenntnis hatte; war dieser in Unkenntnis - ein Fall von dem erst der Schlufsatz des Pr. handele - so sei allerdings beim Spezialmandat die Kenntnis des Herrn zu berücksichtigen. Diese von Cuiacius $^{4}$ ) verteidigte Auslegung haben die meisten angenommen. ${ }^{5}$ )

1) S. 279, 284, 281 N. 368. - 2) Vgl. auch Victor Bruns, Besitzerwerb durch Interessenvertreter 31 N. 2, 3. Der Gedanke des Vertrauensschutzes blitzt flüchtig auf in D. $(44,4) 4,23$ : unde enim divinat is, qui cum tutore contrahit? Doch der Satz ist interpolationsverdächtig. - $\left.{ }^{3}\right)$ So auch Solazzi 4 N.1, der deshalb die Worte et certum emerit für unecht erklärt, ohne jede Erklärung, warum denn die Kompilatoren diese Worte einschoben. Die schweren sonstigen Mängel des Textes hat S. ganz übersehen. - 4) A.a. O. - s) Barth. Chesius S. 476; Glück 20,52; Mühlenbruch 119 f.; anders Westphal, Vom 
Indessen die Beschränkung des negierenden Satzes „scientiam domini non spectandam" auf den Fall der gleichzeitigen Kenntnis des Sklaven hat im Text keine Grundlage und ist auch inhaltlich dem Juristen nicht zuzutrauen. Eine Mahnung bei Kenntnis des Herrn und des Sklaven nur auf die des letzteren, nicht auf die des Herrn zu schauen, ist doktrinär und überflüssig; in solchem Falle ist es wirklich ganz gleichgültig, ob die Person des Vertreters oder die des Vertretenen berücksichtigt wird, das ganze Problem taucht erst auf, wenn nur der eine o der der andere Kenntnis hat. Dieser negative Satz hat nur Sinn, wenn man dabei an den Fall: Kenntnis des Herrn, Unkenntnis des Sklaven denkt. Man darf auch nicht sagen, es handele sich bei dem Schlußsatze um eine modifizierende Note Africans gegenüber der vorangegangenen Julian-Entscheidung, denn wie wir aus Fr. 2 wissen, entspricht gerade der Schlußsatz ganz der Lehre Julians.

$\mathrm{Zu}$ diesen befriedigend nicht $\mathrm{zu}$ behebenden Widersprüchen treten nun noch schwere stilistische Mängel hinzu.

1. Delictum. Die Glosse notiert: stultitiam appellat

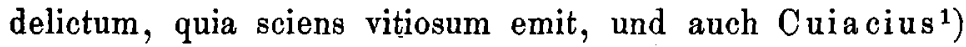
weiß nichts anderes: delictum, id est culpa vel stultitia servi qui sciens emit hominem morhosum vel vitiosum. Dogmatisch muß man so erklären, daß aber nach klassischem Sprachgebrauch hier kein Delikt vorliegt, ist klar; die Kompilatoren aber reden bekanntlich wie alle primitiveren Juristen mit Vorliebe in strafrechtlichen Formeln. ${ }^{2}$ - 2. Tunc. Dieses Wort hat keine Beziehung zum Vorhergehenden. Übersetzt man es mit "in diesem Falle", so ist es überflüssig, denn es ist nur von einem einzigen Falle die Rede; nimmt man es im Sinne von "sodann", so paßt es auch nicht, denn eine andere Begründung ist nicht vorhergegangen. - 3. Ill ud ex bona fide est servum, cum quo negotium sit gestum, deceptum non esse. Dieser Satz läßt sich überhaupt

Kaufe (1791) $\$ 405 \mathrm{a}:$ „Allenfalls könnte man in 1.51 pr. die Worte: sed si servus mandato domino als einen Widerspruch gegen das, was vorher ein anderer anders behauptet, ansehen."

1) A. a. O. Op. 1, 1307. - 2) Vgl. z. B. Beseler, Beitr. z. Kritik der röm. Rechtsquellen $2,16$.

Zeitschrift für Rechtsgeschichte. XXXIII. Rom. Abt. 
nicht übersetzen. Wörtlich übertragen besagt er: „auch das entspricht der bona fides, daß der Sklave nicht getäuscht worden ist", was ganz sinnlos ist. Bedeuten sollen die Worte vielleicht: „jene Entscheidung (daß scientia servi schadet) entspricht auch der bona fides, weil der Sklave nicht getäuscht worden ist", gesagt ist das aber nicht. Schon Cuiacius eilte über diesen Satz mit einer sehr flüchtigen Paraphrase hinweg: ex bona fide esse servum deceptum non esse, id est ignorantiam servi inspici debere. ${ }^{1}$ ) Die Glosse schweigt ganz(!), und so auch die meisten späteren Erklärer. Der letzte gemeinrechtliche Schriftsteller über unsere Lehre, Dunkhase, erklärte, "die Entscheidung werde darauf gestützt, daß nach den Grundsätzen über bona fides der Sklave, welcher den Vertrag geschlossen habe, nicht getäuscht werden dürfe ${ }^{\text {" }}$ ), traute aber dabei African ein eigentümliches Latein zu. -

Schließlich ist auch noch das Prinzip delictum eiusdem (servi), quod in contrahendo admiserit domino nocere debet offensichtlich unrichtig, wie schon ein Blick auf Fr. 2 cit. zeigt.

Einen mit so vielen Mängeln behafteten Text muß auch der vorsichtigste Interpolationenforscher als interpoliert anerkennen; die Ausführung ut nihil-debet trägt durchaus kompilatorisches Gepräge. Sind diese Worte aber Kompilatorenwerk, so läßt sich auch ungefähr erraten, was der Klassiker geschrieben hat. Die Polemik der Kompilatoren wird sich hier wie so oft gegen ihre Vorlage richten, hier werden jene Unterscheidungen gestanden haben, die die Kompilatoren so eifrig ablehnen. Demnach haben Julian-African drei Unterscheidungen erörtert: 1. Pekuliar- und Nichtpekuliargeschäft, 2. beauftragtes und auftragloses Geschäft, 3. Geschäft auf Grund eines Spezial- und eines Generalauftrags (unter die generell aufgetragenen Geschäfte im Sinne dieser Einteilung würden auch die Pekuliargeschäfte zu rechnen sein).$^{3}$ ) Die juristische Bedeutung dieser Unterscheidungen kann nicht zweifelhaft séin. Die zweite ist uns bereits aus Fr. 2 cit. bekannt: bei der beauftragten Vertretung wird aus-

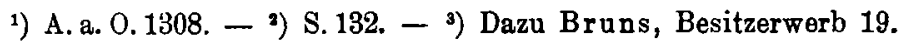


schließlich die Person des Vertretenen berücksichtigt, bei der auftraglosen die des Vertreters; wer die dritte Unterscheidung vertrat, wollte natürlich nur beim certum mandare der Person des Vertretenen Berücksichtigung schenken; die erste Unterscheidung endlich wird uns noch in mehreren Fragmenten begegnen, aus denen man ersehen kann, daß ihre Vertreter beim Nichtpekuliargeschäft die Person des Vertretenen, beim Pekuliargeschäft die des Vertreters maßgebend sein lassen wollten. Für welche dieser drei besprochenen Möglichkeiten sich Julian und African entschieden haben, läßt sich natürlich mit Sicherheit nicht sagen. Der stehen gebliebene echte Schlußsatz des Pr. weist auf die zweite, wozu auch die Entscheidung Julians in Fr. 2 cit. paßt: nach Fr. 2 schadet Kenntnis des Sklaven bei Unkenntnis des Herrn nicht, wenn der Sklave Auftrag hatte; hier erscheint jetzt als Pendant der umgekehrte Fall: Kenntnis des Herrn schadet bei mandierter Vertretung trotz Unkenntnis des Sklaven. - Kann man so noch ungefähr den klassischen Grundtext erkennen, so sieht man andererseits auch recht deutlich aus der Interpolation, was die Kompilatoren wollten: kurzweg Beseitigung der klassischen Differenzierungen, die Kenntnis des Vertreters soll immer schaden; daß auch die Kenntnis des Vertretenen ihrer Ansicht nach schädlich sein soll, die Worte "mandatu domini" des Schlußsatzes im kompilatorischen Sinne nur als beispielsweise Hervorhebung des Mandats zu verstehen sind, läßt sich aus dieser Stelle nicht entnehmen, wohl aber, wie sich zeigen wird, aus andern.

Der $\S 1$ unsres Fragmentes ist völlig unberührt geblieben und macht keine Schwierigkeiten. Der Prokurator ist indirekter Stellvertreter, für die Zuständigkeit der actio redhibitoria in seiner Person ist daher seine Kenntnis und Unkenntnis ausschließlich maßgebend; nur wenn etwa der Procurator seine Klage an den Geschäftsherrn zediert hätte, könnte dessen Kenntnis vom Beklagten entgegengehalten werden. ${ }^{1}$ ) Diese Ausführungen des $\$ 1$, die durchaus klassisches Gepräge tragen, stehen nun aber in Widerspruch mit dem zweiten Fragment, das von der Kenntnis der Sachmängel bei Kauf durch Stellvertreter handelt, nämlich mit

1) Buchka 58 . 
D. $(18,1) 13$ :

Pomponius libro nono ad Sabinum.

Sed si servo meo vel ei cui mandavero vendas sciens fugitivum illo ignorante, me sciente, non teneri te ex empto verum est.

Da man unter "ei cui mandavero" hier den freien Vertreter verstehen muß, so besagt die Stelle, daß die Kenntnis des Vertretenen bei der indirekten Vertretung dem Vertreter vom Drittkontrahenten entgegengehalten werden kann, während Fr. 51 \& 1 cit., wie wir eben sahen, das Gegenteil lehrt. Man hat den Widerspruch geleugnet, meist indem man behauptete, die Stelle rede nur von dem Falle, daß dem Prinzipal die Klage abgetreten worden ist, und er nunmehr gegen den Drittkontrahenten vorgeht. ${ }^{1}$ ) Doch einmal steht in der Stelle von der Klagenzession nichts, andererseits wäre auch dann noch der Gegensatz servus - is cui mandavero unlogisch, ein Gegensatz wie Schimmel und Hengste. Mandry hat vorgeschlagen, das "vel" unsres Textes nicht mit „oder" sondern mit "nämlich", "wenigstens" zu übersetzen, so daß die Stelle nur vom beauftragten Sklaven spricht ${ }^{2}$ ); indes die Stellen, auf die Mandry als Belege für diesen Sprachgebrauch verweist, sind wahrscheinlich alle unecht. ${ }^{3}$ ) So bleibt denn nichts

1) Z. B. Mühlenbruch 122; Buchka 59; Schmid 2, 146; Vangerow, Pand. 3 (ī. Aufl.) $\S 608$ S. 300; Pernice, Labeo 1,503. - Von andern Vereinigungen siehe z. B. die des Vivianus in Casus ad h. l., der den Fall ganz anders drehte: si mandavi alicui, ut servo meo vendat Stichum. ..; D unkhase 139 wollte aus dem vorangehenden Fr. 13 die Worte "me praesente" herübernehmen, historisch unzulässig, da Fr. 12 vom Irrtum, Fr. 13 von der Kenntnis ädilizischer Mängel redet und beide Stellen verschiedenen Werken des Pomponius angehören. Gegen Jherings Auslegung (Jhering J. 1, 290 N. 23) mit Recht Solazzi 9 N.1. - 2) 1,128 N.2. - 3) Man sehe: D. $(17,1) 60,3 \mathrm{Si}$ inter maritum et socerum id actum esset vel tacito intellectu ut rel. Schon van de Water wollte ,intellectum " schreiben, es liegt aber wohl eine flüchtige Interpolation vor, die das sinnlose "actum tacito intellectu " verschuldet hat. - D. $(47,8) 2,24$ si quis igitur interesse sua vel modice docebit rel. Der ganze $\S$ ist bereits von De Ruggiero, Bullettino 19, 69 als unecht erkannt worden. - D. $(48,5) 40,3$ Nupta, priusquam adulter damnetur, adulterii non postulatur, si nuptias denuntiatio vel ad domum mulieris missa non praecessit. Schon Mommsen hat (gegen die Bas.) vel ad iudicem einschieben wollen. - D. $(48,5)$ 
14, 8: Si minor duodecim annis in domum deducta adulterium commiserit, mox apud eum aetatem excesserit coeperitque esse uxor, non poterit iure viri accusari ex eo adulterio, quod ante aetatem nupta commisit sed vel quasi sponsa poterit accusari, ex rescripto divi Severi quod supra relatum est. Der Mann kann in diesem Falle, grade nach dem erwähnten Reskripte (genauer ist es ein Reskr. des Sever. und Autonin., wie $\$ 3$ zeigt), nicht iure mariti klagen, wie Coll. 4,6 beweist: In uxorem adulterium vindicatur iure mariti, non etiam sponsam. Severus quoque et Antoninus ita rescripserunt. Es müßste also an unsrer Stelle korrekt heifen: sed iure extranei poterit accusari; was dasteht, will offenbar sagen, dafs der Manu doch auch in diesem Falle die Vorzugsstellung habe, was unklassisch ist. Die gleiche Interpolation zeigt noch deutlicher C. $(9,9) 7$ : Propter violatam virginem adultam qui postea maritus esse coepit accusator iustus non est et ideo iure mariti crimen exercere non potest, nisi puella violata sponsa eins fuerit. Hier wird direkt behauptet (entgegen der Collatio!), daf der Mann iure viri klagen könne. Der Satz ist auch stilistisch verunglückt: verlobt war das Mädchen mit dem späteren Ehemanne doch auf alle Fälle! Gesagt sein soll, daf das stuprum in die Brautzeit gefallen ist. Demnach wird wohl auch die Mitteilung des Reskripts $D$. $(48,5) 14,3$ verändert worden sein. Mommsen, Röın. Strafrecht 695 hat diese Unstimmigkeiteu nicht erkannt, weil er anscheinend die Collatio-Stelle überseben hatte. - D. $(48,4) 7,4$ : Crimen maiestatis facto vel violatis statuis vel imaginibus maxime exacerbatur in milites. Mom mse $\mathrm{mat}$ auch hier durch Konjektur, aber wider die Basiliken helfen wollen. - C. $(4,65) 1$ : Dominus horreorum periculum vis maioris vel effracturam latronum conductori praestare non cogitur. Coll. 10,9 erweist, daß der echte Text effracturae hatte, vel also im Sinne von oder gebrauchte. D. $(16,3) 1,47$ : an tamen vel de pretio teneatur? Daf die Kompilatoren in diesen $\$$ eingegriffen haben, ist bekannt, vgl. P. Krüger ad h. 1. D. $(21,2) 38$ : In creditore qui pignus vendidit tractari potest, an re evicta vel ad hoc teneatur ex empto, ut quam habet adversus debitorem actionem, eam praestet: habet autem contrariam pigneraticiam actionem. et magis est ut praestet: cui enim non aequum videbitur vel hoc saltem consequi emptorem, quod sine dispendio creditoris futurum est. Den SchluE hat schon Kalb verdächtigt, aber auch vorher ist (anders SeckelHeumann v. vel) nicht alles in Ordnung (statt praestet müfte es praestare debeat beifen; der lehrhafte Satz habet - actionem). - D. $(40,5)$ 24,17: Quid ergo, si plures servos rogatus sit manunittere et ad quorundam pretium sufficiat id quod relictum est, ad omnium non sufficiat, an cogendus sit quosdam manumittere? et putem debere eum cogi vel eos, quorum pretium patitur, manumittere. quis ergo statuet, qui potius manumittitur? utrumne ipse legatarius eligat, quos manumittat, an heres a quo legatum est? et fortassis quis recte dixerit ordinem scripturae sequendum. quod si ordo non pareat, aut sortiri cos oportebit, ne aliquam ambitionis vel gratiae suspicionem praetor subeat, aut meritis 
anderes übrig ${ }^{1}$ ), als auch diesen Text für interpoliert.zu erklären: die Berücksichtigung der Kenntnis des Vertretenen im Rechtsverhältnisse des indirekten Vertreters zum Drittkontrahenten ist ein kompilatorischer Gedanke, wie sich auch noch in einem anderen Fragmente zeigen wird. ${ }^{2}$ ) Stilistisch fällt auf, daß anfangs von zwei Käufern (servo vel eo cui mandavero), im Fortgang nur von einem (illo ignorante) gesprochen wird. ${ }^{3}$ ) Nicht mit der gleichen Sicherheit läßt sich sagen, was Pomponius geschrieben hat. Drei Möglichkeiten sind gegeben: entweder er schloß sich Julian an und schrieb sed si servo meo cui mandavero vendas ${ }^{4}$ ); oder er erklärte, wie auch andere Klassiker, die Kenntnis des Herrn stets für schädlich, mochte der Sklave im Auftrag oder ohne Auftrag gehandelt haben und schrieb dann si servo meo vendas. Schließlich wäre es auch noch möglich, daß Pomponius überhaupt nicht vom Sklaven, sondern vom freien Vertreter sprach, dann hätte der Text gelautet sed si ei cui mandavero vendas sciens fugitivum illo ignorante, me sciente teneri te ex empto verum est. Entscheiden läßt sich hier nichts, sicher ist nur, daß den überlieferten Text Pomponius nicht geschrieben hat.

3. Kenntnis beim Kauf eines Scheinsklaven.

D. $(40,12) 16$ und 17 :

Ulpianus libro quinquagesimo quinto ad edictum.

2. Tunc habet emptor hanc actionem, cum liberum esse nesciret: nam si scit liberum et sic emit, ipse se circumvenit.

cuinsque allegatis arbitrari eos oportet. Die Stelle ist sicher interpoliert: "quorum pretium patitur" ist kompilatorische Breviloquenz. Über fortassis Beseler Beiträge 2,11. Der Gedanke, der Prätor soll nur ja keine Angriffstläche für Angriffe der öffentlichen Meinnng bieten, ist schwerlich klassisch. Arbitrari ist hier sehr seltsam. - Nach diesen Ergebnissen wird natürlich auch D. $(19,2) 10$ verdächtig: ex conducto recte agam vel in hoc ut me liberes. Ganz exakt wird sich die Frage natürlich erst mit dem Vokabular beantworten lassen.

1) Schloßsmanns Vorschlag (Stellvertretung 2, 271), den Widerspruch dadurch zu beseitigen, daß man in fr. 51 \$ 1 das ${ }_{n}$ non ${ }^{4}$ der Schlukentscheidung streicht, ist ganz unannehmbar; vgl. Solazzi 7 f. Schon das „at" fordert eine negative Entscheidung. - 2) Unten S. 77. - ${ }^{3}$ ) Solazzi 9. - 4) Dies vermutet Solazzi a.a. O. ohne weiteres als den echten Text. Aus D. $(21,1) 51$ pr. folgt aber gar nichts. 
3. Quare si filius familias emit, si quidem ipse scit, pater ignoravit, non adquisiit patri actionem: hoc si peculiari nomine egerit. $\left.{ }^{1}\right)$ ceterum si patre mandante, hic quaeritur, an filii scientia noceat: et puto adhuc nocere, quemadmodum procuratoris nocet. flii scientia non nocet, quemadmodum nec procuratoris nocet.

4. Plane si filius ignoravit, pater scit, adhuc dico repellendum patrem, etiamsi peculiari nomine flius emit, si modo pater praesens fuit potuitque filium emere prohibere.

Paulus libro quinquagensimo primo ad edictum. In servo et in eo, qui mandato nostro emit, tale est, ut, si certum hominem mandavero emi sciens liberum esse, licet is cui mandatum est ignoret, idem sit: et non competet ei actio danda mihi actio non est. contra autem, si ego ignoravi, procurator scit, non est mihi deneganda.

Es handelt sich in diesen Fragmenten um die Klage aus dem Ediktstitel: Si quis, cum se liberum esse sciret, dolo malo passus erit, se pro servo venum dari. ${ }^{2}$ ) Es war für diesen Fall eine Strafklage auf den doppelten Kaufpreis gegen den Scheinsklaven verheißen ei, qui eum emerit, cum eum liberum esse nesciret. ${ }^{3}$ ) Natürlich mußte also auch hier die Frage auftauchen: auf wessen Kenntnis kommt es an, wenn ein Vertreter einen Scheinsklaven kauft, auf die des Vertreters oder des Vertretenen?

Vorweg zu bemerken ist dabei, daß diese Klage, auch wenn ein freier Vertreter den Scheinsklaven gekauft hat, doch ohne weiteres dem Vertretenen direkt erworben wird ${ }^{4}$ ); das sagt Ulpian unzweideutig:

$$
\text { D. }(40,12) 22,1
$$

"emere" sic accipiemus, etiamsi per alium quis emerit, ut puta procuratorem.

Natürlich interpretiert Ulpian mit diesen Worten einen Text, nach Lenel ${ }^{5}$ ) den der Formel, und zwar das Stück derselben, das von der Aktivlegitimation zur Klage sprach.

1) Wohl nur ein, allerdings alter, Schreibfehler für emerit. -2) Lenel, Edictum (2. Aufl.) S. 374. - 3) Lenei a.a. O. - *) Das hebt Hupka, Vollmacht S. 65, mit Recht hervor. -- ') A.a. O. S. 374 N. 5 . 
Nunmehr zur Kritik, zunächst des Fr. 16 cit. Hier begegnen wir zunächst den uns schon bekannten Unterscheidungen: Geschäfte peculiari nomine - patris nomine, und mandatu patris - sine mandatu; Ulpian verschränkt freilich die beiden Unterscheidungen ineinander: peculiari nomine - patre mandante, doch ist dies nicht allzu verwunderlich: das mandierte Geschäft ist der Hauptfall der Geschäfte patris nomine, und ihn mußte Ulpian auch deshalb herausgreifen, um die Parallele zum Prokurator ziehen zu können. Die $\S \S 3$ und 4 enthalten im ganzen vier Entscheidungen (in dem etiamsi ... stecken zwei); davon entspricht die erste und dritte durchaus den bisher entwickelten Grundsätzen: beim Pekuliargeschäft schadet die Kenntnis des Sohnes, liegt Mandat vor, so schadet die Kenntnis des Vaters. Die letzte Entscheidung, die beim Pekuliargeschäft auch die Kenntnis des Vaters unter Umständen für schädlich erklärt, hält wenigstens grundsätzlich an Julian fest, der in diesem Falle die Kenntnis des Gewalthabers überhaupt für unbeachtlich erklärte. ${ }^{1}$ ) Die zweite Entscheidung aber, nach der auch beim Abschluß eines aufgetragenen Geschäfts die Kenntnis des Sohnes schädlich sein soll, stellt sich in unversöhnlichen Widerspruch mit D. $(44,6) 2$, die diese Kenntnis nicht beachtet. ${ }^{2}$ ) In der Tat ist diese zweite Entscheidung unseres Fragmentes unecht, Ulpian wird gerade das Gegenteil gesagt und wie Julian in D. $(44,6) 2$ entschieden haben: bei beauftragter Geschäftsführung wird ausschließlich die Kenntnis des Vaters berücksichtigt; das läßt sich wahrscheinlich machen ${ }^{3}$ ):

1. Der ganze Aufbau der Stelle verlangt, daß die zweite Entscheidung umgekehrt lautet als sie überliefert ist. Am Schlusse des $\$ 4$ wird nämlich nicht gesagt: repellendum patrem et si patre mandante et si peculiari nomine filius emit, vielmehr: auch wenn der Sohn peculiari nomine kontrahiert hat (selbstverständlich wenn patre mandante), schadet des Vaters Kenntnis. In diesem "auch wenn" liegt: das, was hier beim Handeln peculiari nomine gesagt wird, kommt

1) Oben S. 42 f. $-{ }^{2}$ ) Oben S. 43, 45. - ${ }^{3}$ ) Verfehlt Solazzi 14: Contro la $1.16 \$ 3$ non si elevano sospetti d'interpolazione. 
nach dem Vorangehenden unerwartet, man möchte auf eine andere Entscheidung gefaßt sein; das ist ja auch ganz richtig, denn wenn - wie im Anfang des $\S 3$ gesagt - die Kenntnis des Sohnes beim Pekuliargeschäft immer schadet, möchte man meinen, daß seine Unkenntnis immer nützen müßte. Dagegen kommt dem Schreiber offenbar gar nicht überraschend die Entscheidung: bei mandiertem Handeln nützt die Unkenntnis des Sohnes nicht, also muß im Vorangehenden gestanden haben, daß seine Kenntnis auch nicht schadet.

2. Es ist absurd, erst eine Entscheidung zu fällen (non adquisiit actionem), diese sofort ausdrücklich auf die Pekuliargeschäfte zu beschränken, fortzufahren „wenn aber der Vater Auftrag gab" und dadurch im Leser die Erwartung hervorrufen, jetzt komme die entgegengesetzte Entscheidung - und dann doch diesen Fall genau so wie den vorhergehenden zu entscheiden. Wenn Ulpian sagen wollte: beim Pekuliargeschäft ist meine Entscheidung zweifellos, beim aufgetragenen Geschäft fraglich (hic quaeritur) ${ }^{1}$ ), so hätte er schreiben müssen: hoc, si peculiari nomine emerit, nunquam in dubium venit, nicht aber das, was dasteht.

3. Die beiden aufeinanderfolgenden "adhuc" sind unschön, unschön auch der abgerissene Stil, der die Subjekte trotz Kasuswechsel nicht wiederholt: puto (eam) nocere quemadmodum procuratoris (scientia) nocet.

4. Der verdächtige Satz widerspricht endlich der sicher echten Schlußentscheidung des Fr. 17; in dieser heißt es: si ego ignoravi, procurätor scit, non est mihi deneganda, Kenntnis des Prokurator schadet also nicht, umgekehrt sagt unser § 3: procuratoris scientia nocet. Man hat auch diesen Widerspruch bisweilen geleugnet: Ulpian rede von einem generellen, Paulus von speziellem Auftrag ${ }^{2}$ ); doch in der Ulpianentschei-

1) So wollte die Stelle verstehen Mitteis, Stellvertretung 286 N. 375. - ${ }^{2}$ ) So schon Gl. Procuratori zu Fr. 17, Cuiacius ad Fr. 17 (Comment. ad libros Pauli ad ed. Op. 8,671 Mutinae 1780). Von Neueren Dunkhase 120. Leonhard 126 hält fest an der von Hellmann 160 verteidigten Auslegung: „Die Worte quemadmodum - nocet bedeuten 'unter denselben Voraussétzungen, unter denen die Kenntnis des Prokurator schadet' d. h., wie aus 1.17 hervorgeht, bei einem Generalmandat, nicht bei einer besonderen Weisung." Dies ist eine dogma- 
dung findet sich diese Beschränkung nicht. In der Literatur des jüngsten gemeinen Rechts hat man aber den Widerspruch meist zugegeben und eine Meinungsverschiedenheit zwischen Ulpian und Paulus angenommen. ${ }^{1}$ ) Die Entscheidung des Fr. 17, an der man sehr mit Unrecht mit kühnen wider die Basiliken ${ }^{2}$ ) streitenden Konjekturen herumgebessert hat ${ }^{3}$ ), ist aus einem sogleich zu erörternden formellen Grunde als die echte zu erachten, sie ist aber auch die sachlich korrekte ${ }^{4}$ ), unsern bisher entwickelten Prinzipien, insbesondere D. $(44,6) 2$ entsprechend: wobei man sich vergegenwärtigen muß, daß der freie Prokurator in Ansehung dieger prätorischen Strafklage genau so wie der gewaltunterworfene Vertreter dasteht, da auch er, wie oben gesagt ${ }^{5}$ ), diese Klage direkt seinem Mandanten erwirbt.

Aus allen diesen Gründen ist der $\S 3$ des Fr. 16 für interpoliert zu erklären. Die Kompilatoren sind hier genau so vorgegangen wie im Fr. 51 21, 1: die mandierte und die nicht mandierte Vertretung, das Pekuliargeschäft und das Geschäft nomine patris vel domini sollen gleich behandelt werden, die Kenntnis des Vertreters soll immer schaden:

tische, historisch aber unhaltbare Vereinigung. Die Übersetzung ist sprachlich unmöglich, quemadmodum kann die postulierte Bedeutung nicht haben. Der von Hellmann und Leonhard verlangte Gedanke hätte lateinisch etwa ausgedrückt werden müssen: et puto scientiam eo casu nocere, quo procuratoris scientia noceret. Außerdem trauen diese beiden Schriftsteller Ulpian eine eigentümliche Entscheidung zu: den vorliegenden Fall entschiede er gar nicht, sondern verwiese auf einen analogen, für den er aber auch keine Entscheidung gibt. Bereits Mitteis 285 N. 375 hat die Auslegung Hellmanns abgelehnt. - Eine andere nicht minder verfehlte Vereinigung bei Puchta, Vorlesungen (5. Aufl.) 2, $117 \$ 275$.

1) So Curtius 91; Rudorff in seiner Note zu der zitierten Stelle aus Puchtas Vorlesungen; Vangerow $3 \S 608$ S. 300; Mitteis, Stellvertretung 285 N. 375; Hupka, Vollmacht 65 und jetzt wieder

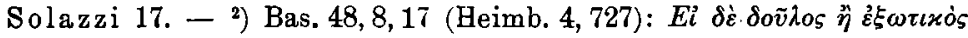

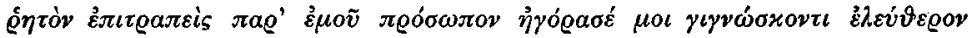

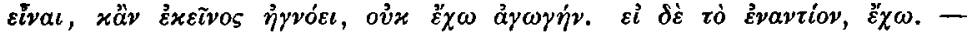
8) Mühlenbruch 126f.; Buchka 14 ff. 60; Schmid 2, 141,399. Siehe auch Schulting-Smallenburg, Notae 6,529. - 4) Richtig bemerkt von Hupka, der aber doch auch die Entscheidung Olpians für echt hält. - Б) Oben S. 55. 
diese inhaltliche Verwandtschaft unsres $\& 3$ mit dem als sicher unecht erwiesenen Teile von Fr. 51 setzt den Schlußstein auf unsern Nachweis, dab auch unser $\$ 3$ interpoliert ist. ${ }^{1}$ )

Auch das, in seinem Schlußsatz soeben schon benutzte Fr. 17 ist stark interpoliert.

1. et non competet ei actio. Hier ist zunächst das "ei $i^{*}$ falsch, es müßte "mihi" dastehen, wie auch schon die Verfasser der Basiliken korrigiert haben. ${ }^{2}$ ) Vielfach hat man allerdings die überlieferte Lesart verteidigt, so noch neustens Leonhard ${ }^{3}$ ); doch man erhält dann den Satz, daß die Kenntnis des Prinzipals dem freien Prokurator in seinem Verhältnis zum Drittkontrahenten schädlich ist, was der sicher echten Entscheidung des Fr. 51 \$ 1 D. 21,1 widerspricht. Leonhard hat freilich diesen Widerspruch ganz erklärlich gefunden, die beiden Fälle seien eben sehr verschieden: in dem einen (Fr. 51,1) werde ein mit Enttäuschungen verbundener Vertrag aufgehoben, in dem andern (Fr. 17) werde ein Delikt mit Nachteilen verknüpft. Dabei übersieht aber Leonhard, daß die klassische actio redhivitoria, von der Fr. 51 spricht, gar nicht auf Aufhebung des Vertrages geht, vielmehr genau so eine amtsrechtliche Strafklage aufs Doppelte ist $^{4}$ ) wie die Klage gegen den Scheinsklaven.

1) Den $\$ 4$ unsres Fragments haite ich für echt. G. Beseler, Beiträge 2, 36 erklärt bei Gelegenbeit der Kritik von $(2,15)$ 8, 23: „Dico ist für Ulpian zu selbstbewufit"; es könnte schon sein, daf auch hier die Kompilatoren dieses Wort erst eingesetzt hätten, um gegenüber dem von ihnen geschriebenen puto etwas Abwechselung zu schaffen. - ${ }^{2}$ ) Oben S. 58 N. 2. - ${ }^{3}$ ) S. $126-128$, ebenso Dunkhase 122. - ${ }^{4}$ ) Das sollte man wirklich nicht mehr bestreiten; vgl. Lenel, Edictum 531 f. (2. Aufl.); Peruice, Labeo 2,2,1 (2. Aufl.) S. 50-53. Freilich so ganz untadelhaft, wie Lenel glaubt, ist der entscheidende Quellenbeleg nicht: D. $(21,1) 45$ Redhibitoria actio duplicem babet condemnationem: modo enim in duplum modo in simplum condemnatur venditor. nam si neque pretium neque accessionem solvat neque eum qui eo uomine obligatus erit liberet, dupli pretii et accessionis condemnari iubetur: si vero reddat pretium et accessionem vel eum qui eo nomine obligatus est liberet, simpli videtur condemnari. Verdacht bezüglich des Schlußssatzes hat schon Pernice Labeo 2,2,1 S. 249 N. 6 geäufert, m. E. ist er sicher interpoliert, besagt er doch, dafs derjenige, der gutwillig den Preis zurückgibt, auf eben diesen verurteilt zu sein scheint, ein Gedanke, der dem Klassiker nicht zuzutrauen ist. Der Nachweis Ecks, 
Das "ei" ist also unrichtig. Es ist aber keineswegs auf ein Schreiberversehen, sondern auf Interpolation zurückzuführen, denn gerade in diesem Satze ist die Hand der Kompilatoren unzweideutig zu erkennen, nämlich an dem Worte "competet". Der Klassiker kann nicht in demselben Satze competet actio - deneganda est actio gesagt haben; wenn er von der Denegation sprach, muß er auch von dare actionem und nicht von competere gesprochen haben: die Kompilatoren haben wie so oft competere statt dare eingesetzt $^{1}$ ), dabei die Konstruktion vergessen und das falsche ei geschrieben.

2. Die Stelle behauptet, Rechtsgeschäfte von Sklaven und Mandataren würden in dieser Frage ganz gleich behandelt. Nach klassischem Recht ist das, wie wir aus dem rekonstruierten Fr. 16 wissen, falsch; auch fällt wieder der unlogische Gegensatz Sklaven und Beauftragte auf. ${ }^{2}$ ) Paulus wird wie Ulpian zunächst von Käufen durch Gewaltunterworfene gesprochen und als Paradigma den Sklaven wie Ulpian den Haussohn gebraucht haben; dann wandte er sich zur Stellvertretung durch Freie. Er scheint nun unterschieden zu haben, ob ein Madatum certum vorlag oder nicht, die Kompilatoren aber wollten, wie wir ja schon wissen, alle derartigen Unterscheidungen beseitigen und haben dies auch wirklich in einem Satze ausgesprochen, der schon durch seine ungewöhnliche Plumpheit auf fällt: „Beim Sklaven und Mandatar ist es so, daß wenn ich bestimmten Auftrag gegeben habe, es dasselbe ist: und mir wird nicht die Klage zustehen." Das soll heißen: beim Kontrahieren von Sklaven und bestimmt Beauftragten schadet in gleicher Weise stets die

Festgabe für Beseler S. $196 \mathrm{f}$, dafa die Klassiker auch das arbitrium bisweilen untechnisch condemuatio nennen, ist völlig miflungen. Die ,am deutlichsten" redende Stelle D. $(21,1) 25,5$ ist interpoliert (Grünhut 38,50$)$, die übrigen beweisen nichts. Auch fordert der ganze Aufbau der Stelle statt videtur: iubetur. Grade diese Interpolation erweist aber die Ricbtigkeit der Ansicht Lenels: Gaius hatte sie auch unzweideutig ausgesprochen, die Kompilatoren aber wollten nur dem Ungehorsamen die Strafe zudiktieren.

1) Vgl. P. Krü ger, Savigay-Zèitschr. 16,1ff. - ${ }^{2}$ ) Schon die Basilikenverfasser haben dies bemerkt und richtiger formuliert, siehe oben S. 58 N. 2. 
Kenntnis des Vertretenen; was aber der Text sagt, ist unzusammenhängend gestammelt, nicht gesprochen. $\mathrm{DaB}$ die Eingangsworte angeflickt sind, sieht man auch daraus, daß im Fortgang der Stelle nur noch vom Prokurator, nicht mehr vom Sklaven gesprochen wird. Schon Mommsen ist der ungefüge Satz, den aber so schon die Basilikenverfasser gelesen haben, aufgefallen, Hupka hat ihn mit Recht, aber ohne Angabe von Gründen für interpoliert erklärt. ${ }^{1}$ )

Das Fr. 17 zeigt uns demnach, daß die Kompilatoren nicht nur stets die Kenntnis des Vertreters, sondern auch umgekehrt die des Vertretenen schädlich sein lassen wollten ${ }^{2}$ ); was das klassische Recht anlangt, so zeigt das Fragment augenscheinlich Paulus als Anhänger der Unterscheidung: Geschäfte auf Grund eines Mandatum certum und Geschäfte ohne ein solches; daß diese Unterscheidung von manchen Juristen vertreten wurde, dafür gab uns bereits D. $(21,1) 51$ pr. eine Andeutung. ${ }^{3}$ )

Auf Grund dieser m. E. ziemlich sicheren Ergebnisse ist nunmehr Stellung zu nehmen gegenüber

$$
\text { D. }(40,12) 22,4 \text { u. } 5 \text { : }
$$

Ulpianus libro quinquagensimo quinto ad edictum.

4. Heredi et ceteris successoribus ${ }^{4}$ ) scientia sua nihil nocet, ignorantia nihil prodest.

5. Sed si per procuratorem scientem quis emerit, ei nocet, sicuti tutoris quoque nocere Labeo putat.

Die Entscheidung des $§ 5$ widerspricht dem als echt erwiesenen Teil des Fr. 17, stimmt aber überein mit dem unechten Teil von Fr. $16 \S 3$ und wird dadurch selbst verdächtig. Bedenklich ist auch der höchst saloppe Stil: in beiden Halbsätzen fehlt das Subjekt (scientia), das auch keineswegs aus dem vorausgehenden Satz ohne weiteres ergänzt werden kann, da in diesem von scientia und ignorantia gesprochen wird; und selbst wenn man zu ,ei nocet" scientia hinzuzudenken geneigt ist, beim Wechsel des Kasus (tutoris

1) Vollmacht 65 N. 2. Hupka schliekt sich an Solazzi 13, der aber bekennt: oscura è invece la causa del mutamento (S. 14). $\left.{ }^{2}\right)$ Eine gleichartige Interpolation siehe S. 54, 77. $-{ }^{3}$ ) Oben S. $50 \mathrm{t}$. - 4) Longo, Bullettino 14, 151; Mitteis, Röm. Privatrecht 1, 105 N. 28. 
scientiam nocere putat) hätte das Subjekt wiederholt werden müssen. Auch dieses Fragment wird daher unecht sein.

4) Kenntnis beim Erwerb des Ersitzungsbesitzes.

Der Erwerb des Ersitzungsbesitzes setzt auf der Seite des Erwerbers voraus 1. die Kenntnis vom Erwerbe des Besitzes, 2. den Glauben, zum Besitze befugt zu sein. In wessen Person müssen beim Besitzerwerb durch Stellvertreter diese psychologischen Tatsachen gegeben sein?

Was das klassische Recht betrifft, so ist ein $Z_{w}$ eifel bei der Beantwortung dieser Fragen nicht möglich. Die Klassiker unterscheiden zunächst bezüglich der Kenntnis vom Besitzerwerb, ob ein Pekuliargeschäft vorliegt oder nicht: ist der Besitz peculiari nomine erworben, so genügt die Kenntnis des Gewaltunterworfenen, wenn nomine domini vel patris, so ist die Kenntnis des Gewalthabers erforderlich. So zunächst ausdrücklich Paulus und Papinian:

\section{D. $(41,3) 47$}

Paulus libro tertio ad Neratium.

Si emptam rem mihi procurator ignorante me meo nomine adprehenderit, quamvis possideam, eam non usucapiam, quia ut ignorantes usuceperimus, in peculiaribus tantum rebus receptum est.

$$
\text { D. }(41,3) 44,7
$$

Papinianus libro vicensimo tertio quaestionum.

Si, cum apud hostes dominus aut pater agat, servus aut filius emat, an et tenere ${ }^{1}$ ) incipiat? Si quidem ex causa peculii possedit, usucapionem inchohari nec impedimento domini captivitatem esse, cuius scientia non esset in civitate necessaria. Si vero non ex causa peculii comparetur, usu non capi . .

Das gleiche wird uns von Paulus als Lehre des Celsus und des Pomponius bezeugi, wobei wir auch erfahren, daß

1) Man erwartet, wie P. Krüger richtig notiert, an usucapiatur simul atque servus vel filius tenere incipiat; da auch im folgenden der Acc. c. Inf. ohne Verbum ist, so ist ein kürzender Eingriff der Kompilatoren wahrscheinlich: auch im benachbarten $\$ 4$ (vgl. Beseler, Beiträge 2,90) und wohl auch im $\$ 5$ ist die Hand der Kompilatoren bemerkbar. 
die gleiche Unterscheidung hinsichtlich des Erfordernisses der bona fides gemacht wurde.

$$
\text { D. }(41,4) 2,10-13 \text {. }
$$

10. Si servus tuus peculiari nomine emat rem, quam scit alienam, licet tu ignores alienam esse, tamen usu non capies. si tuo nomine emat, usucapies.

11. Nam et Celsus scribit, si servus meus peculiari nomine apiscatur possessionem, id etiam ignorantem me usucapere: quod si non peculiari nomine non nisi scientem me: et si vitiose coeperit possidere, meam esse vitiosam possessionem, si peculiari nomine adquirat possessionem...

12. Pomponius quoque in his, quae nomine domini possideantur, domini potius quam servi voluntatem spectandam esse ait: quod si peculiari, tunc mentem servi quaerendam.

13. Si servus bona fide emerit peculiari nomine ego ubi primum cognovi sciam alienam, processuram usucapionem Celsus ait: initium enim possessionis sine vitio fuisse: sed si eo tempore quo emit, quamquam id bona fide faciat, ego alienam rem esse sciam, usu me non capturum.

So sicherlich echt die in diesem Fragmente überlieferten Sätze sind, so ist doch auch hier die Hand der Kompilatoren bemerkbar, nämlich in dem, was nicht im Texte steht, und was die Redaktoren gestrichen haben müssen. Paulus schreibt „Pomponius quoque“, Celsus muß also der gleichen Ansicht wie Pomponius gewesen sein; Celsus macht nun in der Tat im $\S 11$ die Unterscheidung zwischen Geschäften peculiari nomine und domini nomine wie Pomponius, er benutzt aber nach dem überlieferten Text diese Unterscheidung nicht zur Beantwortung der Frage, wessen Person in Ansehung der bona fides maßgebend sei, er behauptet vielmehr ganz allgemein, daß der böse Glaube des Sklaven dem Herrn stets (auch beim Erwerb domini nomine) schade, der bösgläubige Besitz des Sklaven (diesen meint Celsus hier mit den Worten vitiosa possessio) ${ }^{1}$ ) stets ein solcher auch des

1) Nicht den gewaltsam oder heimlich erworbenen Besitz, wie die Glosse und Spätere (Mühlenbruch 116) annahmen; das geht unwiderleglich aus $\$ 13$ hervor, wo Celsus mit den Worten "sine vitio" nur die bonae fidei possessio meinen kann. 
Herrn sei. Daß Celsus aber nicht dieser Ansicht war, zeigt der § 13, wo es auch in Ansehung der bona fides zwischen Pekuliargeschäft und Geschäften domini nomine scheidet. Die Kompilatoren müssen also die Worte des Celsus gestrichen haben, die die Entscheidung si vitiose coeperit possidere, meam vitiosam esse possessionem auf den Fall des Pekuliarerwerbs beschränkten: die Kompilatoren wollten eben auch hier die klassischen Unterscheidungen beseitigen. Man beachte nun auch, daß zwischen $\S 10$ und $\$ 11$ eine Lücke ist; so abrupt wird Paulus schwerlich geschrieben haben, man erwartet ein "nam et Celsus“. Eine längere Erörterung kann andererseits wegen des engen Zusammenhanges $\mathrm{zwischen}$ den beiden $\S \S$ nicht gestanden haben. Wahrscheinlich werden die Kompilatoren hier genau dasselbe gestrichen haben wie im $\S 11$, Paulus wird fortgefahren haben: „si non peculiari nomine emat usucapies"; man erwartet die Erledigung dieses Falles unbedingt. Die Kompilatoren aber strichen auch hier aus den soeben angegebenen Gründen, dabei sind dann wohl die Anschlußworte "nam et" mit verloren gegangen.

Genau dieselbe Interpolation findet sich

$$
\text { D. }(41,3) 43,1
$$

Papinianus libro vicensimo secundo quaestionum.

Patrem usu non capturum, quod filius emit, propter suam vel filii scientiam certum est.

$$
\text { D. }(6,2) 7,13 \text { : }
$$

Ulpianus libro sexto decimo ad edictum.

Sed enim si servus meus emit, dolus eius erit spectandus, non meus, vel contra.

Beide Stellen, von denen die zweite vom publizianischen Besitze handelt, unterscheiden nicht zwischen Erwerb peculiari und domini nomine und erweisen sich durch diesen Widerspruch mit Fr. 2 cit. als unecht. Im Fr. 7 verrät sogar auch der Stil die Kompilatoren: das liederliche „vel contra" kann der Klassiker nicht geschrieben haben; was da steht, heißt ja wörtlich: der Dolus des Sklaven ist zu beachten, nicht der meinige oder umgekehrt (demnach mein dolus, nicht der des Sklaven); was ist denn dann Rechtens? (iemeint haben die Kompilatoren wohl mit den Worten ${ }^{\text {vel contra }}$ " wie 
schon die Glosse erklärte: bona fides eius non mea spectanda est, doch solche saloppe Breviloguenz ist echt kompilatorisch.

5. Die Kenntnis bei der Passivlegitimation zum interdictum quod vi aut clam.

D. $(43,24) 21,1$ :

Pomponius libro vicensimo nono ad Sabinum.

Si iussero servum meum opus facere, cum quantum ad me portinet, in clandestini suspicionem non veniret, servus autem meus putaverit, si rescisset adversarius, prohibiturum eum, an tenear? et non puto, cum mea persona sit intuenda.

Die Passivlegitimation zum interdictum quod vi aut clam setzt, wenn es sich um heimliche Eingriffe handelt, voraus, daß der Täter gegen sein Tun Einsprache fürchtete oder fürchten mußte, daß er also den animus celandi habe. Wie nun, wenn ein Gewaltunterworfener den Eingriff begeht, der den animus hat, während er beim Gewalthaber fehlt? Handelte der Gewaltunterworfene ohne Auftrag, so schadet sein animus dem Herrn, denn dieser haftet noxal ${ }^{1}$ ); hat er im Auftrage des Gewalthabers gehandelt, so kommt sein animus überhaupt nicht in Betracht, der Gewalthaber haftet nicht, nicht einmal noxal. ${ }^{2}$ )

Die Entscheidung entspricht durchaus klassischen Grundsätzen, interpoliert ist sie wohl nur zur Verdeutlichung; der ungeschickte Satz quantum-meus, der einen einfachen Gedanken in geschraubtester Form ausspricht, ist jedenfalls schwerlich dem Klassiker zuzuschreiben. ${ }^{3}$ )

1) Lenel, Edictum 465. - 2) Die Glosse Intuenda behauptet freilich das Gegenteil: "sed nomine servi tenear ut supra eod. l. nam et si servus $(43,24,14)^{\star}$; doch dort wird von einem Sklaven gesprochen, der ohne Auftrag handelt. Der Klassiker könnte auch nicht søgen „non tenear", wenn. doch noxaliter gehaftet würde, das wäre doch eben auch eine Haftung. - a) $\mathrm{Zu}$, in suspicionem venire" siehe ${ }_{n}$ ad suspicionem adducere ${ }^{\prime}$ in dem gleichfalls interpolierten D. $(9,2) 40$ : In lege Aquilia, si deletum chirographum mihi esse dicam, in quo sub condicione mihi pecunia debita fuerit, et interim testibus quoque id probare possim, qui testes possunt non esse eo tempore, quo condicio exstitit, et si summatim re exposita ad suspicionem iudicem adducam, debeam

Zeitschrift für Rechtsgeschichto. XXXIII. Rom. Abt. 
6. Kenntnis beim Erbschaftserwerb.

$$
\text { D. }(29,2) 30,7 \text { : }
$$

Ulpianus libro octavo ad Sabinum.

Scientia autem vel opinio, si filius familias vel servus instituti sunt, utrum ipsorum an domini vel patris accipienda sit? finge patrem putasse praegnatem, filium certum esse fingere et sic adire, an adquirat hereditatem? puto adquirere: sed contra non adquirere.

Ulpian interpretiert hier den in $\S 1$ überlieferten SabinusSatz ${ }^{1}$ ): proximus a filio postumo heres, dum mulier praegnas est aut putatur esse, adire hereditatem non potest; sed si scit non esse praegnatem, potest. Natürlich taucht auch hier die Frage auf: auf wessen Kenntnis kommt es an, wenn dieser "proximus" ein Gewaltunterworfener ist? Die Antwort muß hier notwendig anders lauten als in den bisher betrachteten Fällen. Der Erbschaftserwerb bedarf zwar der Ermächtigung des Gewalthabers, andererseits aber kann dieser nicht selbst die Erbschaft antreten, es bedarf eines gültigen Erwerbsaktes des Gewaltunterworfenen, mindestens seiner Zustimmung. ${ }^{2}$ ) Während daher in den früheren Fällen der Gewalthaber das dem Unterworfenen aufgetragene Geschäft selbst hätte vornehmen können, und die Juristen daher die Person des Unterworfenen ignorieren und allein die Kenntnis des Gewalthabers berücksichtigten durften, müssen hier die Gültigkeitsvoraussetzungen jedenfalls in der Person des Unterworfenen vorhanden sein, und es könnte sich nur fragen, ob noch außerdem die Kenntnis des Gewalthabers nötig sei. Unser Fragment verneint diese Frage; ausschließlich auf die Kenntnis des Sohnes und Sklaven soll es ankommen, aber

vincere: sed tunc condemnationis exactio competit, cum debiti condicio extiterit: quod si defecerit, condemnutio nullas vires habebit. Die Kompilatoren haben gar nicht bedacht, daß Paulus ja einen Fall vor Augen hat, in dem wegen der zerstörten Urkunde nach Eintritt der Bedingung (quo condicio extitit! im Sinne der Kompilatoren müfte extatura est dastehen) geklagt wird.

1) Meine Sabinus-Fragmente in Ulpians Sabinus-Kommentar S. 25. - ${ }^{2}$ ) Leist, Bonorum Possessio 2, 2 S. $123 \mathrm{f}$;; Glück, Serie der Bücher 37, 38 Bd. 2 S. $167 \mathrm{f}$. 
freilich ist die Hand der Kompilatoren unverkennbar: unschön ist die Wiederholung des Wortes fingere in ganz verschiedener Bedeutung (finge: nimm an; fingere: simulieren); zu fingere fehlt das unbedingt nötige Subjekt eam; sed contra non adquirere ist unerträgliche Breviloquenz. Was der Klassiker geschrieben hat, wissen wir nicht. -

Ist zur bonorum possessio ein Hausunterworfener berufen, so frägt es sich, auf wessen Kenntnis es für den Lauf der Agnitionsfrist ankommt, auf die des Sohnes oder des Vaters? Da der Vater die bonorum possessio nicht ausschlagen kann, so kann auch seine Kenntnis allein die Fristen nicht in Lauf bringen $\left.{ }^{1}\right)$, und das sagt auch ausdrücklich

$$
\text { D. }(38,15) 3 \text { : }
$$

Paulus libro quadragensimo quarto ad edictum.

Circa tempora bonorum possessionis patris scientia filio non nocet.

Die Person des Vaters wird nur insofern berücksichtigt, als die Frist nicht in Gang kommt, solange der Sohn nicht in der Lage ist, den Vater von der Delation zu benachrichtigen. ${ }^{2}$ )

7. Dolus bei der prozessualen und außerprozessualen Vertretung.

$$
\text { D. }(44,4) 11 \text { : }
$$

Neratius libro quarto membranarum.

Si procurator cognitor agit, de dolo eius excipi non debet, quia aliena lis est isque rei extraneus, neque alienus dolus nocere alteri debet. Procurator a utem si post litem contestatam dolo quid fecerit, an exceptio eo nomine in iudicum obicienda sit, dubitari potest, quia litis contestatione res procuratoris fit eamque suo iam quodammodo nomine exequitur. et placet de procuratoris dolo excipiendum esse. idem de tutore, qui pupilli nomine aget, dicendum est.

1) Leist bei Glück, Serie der Bücher 37,38 Bd. 3 S. 8. - ') So (D. 38, 15) 5 ; freilich ist die Stelle wohl stark interpoliert: fingamushabebit, sed-comprobasset dürfte Einschub sein. 
1. In universum autem haec in ea re regula sequenda est, ut dolus omnimodo puniatur, etsi non alii cui, sed ipsi, qui eum admisit, damnosus futurus erit.

Man hat bislang noch nicht erkannt, daß auch dieses Fragment von den Kompilatoren verändert worden ist.

Zunächst ist der $\S 1$ unecht; schon Mommsen hat ihn beanstandet und durch Konjektur heilen wollen. Der Satz ist in der Tat barock: der dolus wird immer gestraft, auch wenn er keinem andern als dem Täter zum Schaden gereicht; das soll heißen: auch wenn dem Prozeßvertreter sein Dolus nicht im Prozesse entgegengehalten werden kann, weil das eine Schädigung des Vertretenen bedeuten würde, so bleibt der dolus deshalb doch nicht ungeahndet. Da schon die Verfasser der Basiliken den Satz wie wir heute gelesen haben ${ }^{1}$ ), so ist eine Interpolation wahrscheinlicher als ein Textverderb durch A bschreiber: die Kompilatoren glaubten wieder einmal einschärfen zu müssen, nur ja die actio doli nicht zu vergessen, genau so, wie sie es in der bekannten Papinianentscheidung getan haben $\left.{ }^{2}\right)$ :

\section{D. $(46,3) 95,1$ :}

... nullo modo ex stipulatu agi poterit, cum illo in tempore, quo moriebatur, non commiserit stipulationem. sane quoniam impunita non debent esse admissa, doli actio non immerito desiderabitur.

Viel wichtiger ist freilich die Interpolation des Pr. Hinter "debet" ist hier zweifellos eine Lücke, man erwartet mindestens ein "sed". Aber auch dann wäre die Ausführung noch eigentümlich: im Anfang wird gesagt, für den procurator sei der Prozeß eine res aliena, gleich darauf aber das Gegenteil, res procuratoris fit. Man wende auch nicht ein, daß ja erst mit der litis contestatio res procuratoris fit, denn der Widerspruch bleibt trotzdem bestehen, da der Eingangssatz keine Beschränkung auf die Zeit vor der litis contestatio enthält. Es wäre möglich, daß dieser erste Satz von einem anderen

1) Bas. (51,4) 11 Heimb. 5, 99: Regulariter enim dicitur, omnem dolum esse puniendum, etiamsi non alius, sed ipse qui fecit, damnum. sit passurus. - ${ }^{2}$ ) Uber die Interpolation dieser Stelle Litten, Festgabe f. Güterbock S. 276 ff.; Beseler, Beiträge 2, 16. 
Juristen stammt und die Kompilatoren das Zitat gestrichen haben, für wahrscheinlicher aber halte ich folgendes. Der Eingangssatz ist vollkommen richtig für den cognitor, denn dieser ist auch nach der litis contestatio nicht dominus litis. Koschaker hat mit Recht hervorgehoben, daß die Frage: kann der dolus des Prozeßvertreters diesem mittelst Einrede entgegengehalten werden? für den cognitor schlechthin hätte verneint werden müssen ${ }^{1}$ ); nun, diese für den cognitor passende Entscheidung steht genau in dem Eingangssatz und schon darum ist es wahrscheinlich, daß der "procurator" hier interpoliert ist. Entscheidend spricht aber für diese Interpolation der Satz "neque alienus dolus nocere alteri debet": dieses Argument paßt für den procurator der Zeit des Neratius überhaupt nicht. Der procurator dieser Zeit kann dem Vertretenen gar nicht schaden: auch wenn der dolus des procurator geltend gemacht werden könnte und zur Klagabweisung führen würde, so könnte der dominus einfach noch einmal seinerseits klagen. ${ }^{2}$ ) Dagegen trifft dieses Argument für den cognitor allerdings zu: griffe ihm gegenüber die exceptio doli wegen seines dolus durch, so wäre allerdings der Vertretene durch fremden dolus geschädigt, denn seine Klage ist durch den cognitor konsumiert. Freilich darf man nun nicht etwa auch im zweiten Satze des Pr. den cognitor an die Stelle des procurator setzen ${ }^{3}$ ), Neraz stellte vielmehr beide einander gegenüber. Damit wird auch der Schlußsatz vollkommen klar: placet de procuratoris dolo excipiendum esse. Auf "procuratoris" liegt der Ton, was bei unserer Rekonstruktion wohl verständlich ist. In dem überlieferten Text stellt Neraz nicht cognitor und procurator, sondern dolus des procurator vor und nach der litis contestatio

1) Translatio indicii S. 121 N. 2. - ${ }^{2}$ ) $\mathrm{Da} 3$ der Prokurator dieser Zeit noch keine Konsumptivmacht hatte, ist herrschende Lebre; vgl. Rosenberg, Stellvertretung im Prozef S. 247, hier auch die Literatur über die Frage. - ${ }^{3}$ ) Dies wollte Debray, De la représentation en justice par le cognitor, Thèse (Paris) 1892 S.151 N.2; dagegen mit Recht Koschaker a. a. O.; Wirbel, Cognitor 125 N. 1. Gegen D. spricht entscheidend die Parallele, die Neraz zum tutor zieht, denn dieser hatte zu jener Zeit keine Konsumptionsmacht: Gaius 4, 99 und dazu Rosenberg S. 346, 349. 
gegenüber, man erwartet daher auch als Schlußentscheidung etwa: placet de dolo, quem procurator post litem contestatam admisit, excipiendum esse.

Geradezu barbarisch zugerichtet haben die Kompilatoren

D. $(44,4) 4,17-19$ :

Ulpianus libro septuagensimo sexto ad edictum.

17. In hac exceptione et de dolo servi vel alterius personae iuri nostro subiectae excipere possumus et de eorum dolo, quibus adquiritur. sed de servorum et filiorum dolo, si quidem ex peculiari eorum negotio actio intendatur, in infinitum exceptio obicienda est: si autem non ex peculiari causa, tum de eo dumtaxat excipi oportet, qui admissus sit in ipso negotio quod geritur, non etiam si postea aliquis dolus intervenisset dolo domini dumtaxat excipi oportet: neque enim esse aequum servi dolum amplius domino nocere, quam in quo si opera eius esset usus.

18. Quaesitum est, an de procuratoris cognitoris dolo, qui ad agendum tantum datus est, excipi possit. et puto recte defendi, si quidem in rem suam procurator cognitor datus sit, etiam de praeterito eius dolo, hoc est si ante acceptum iudicium dolo quid fecerit, esse excipiendum, si vero non in rem suam, dolum praesentem in exceptione conferendum de dolo eius excipi non posse. si autem is procurator sit, cui omnium bonorum administratio concessa est post litem contestatem dolo quid fecerit, tunc de omni dolo eius excipi posse Neratius scribit.

19. Mandavi Titio, ut a te stipularetur, deinde Titius Seio, et stipulatus a te Seius est et iudicium edidit: ait Labeo excipiendum esse tam de meo quam et Seii dolo.

Beginnen wir mit der Kritik des $\$ 18$, da auch dieser von der Prozeßvertretung handelt und Neratius zitiert wird. Was in diesem § über den ProzeBprokurator gelehrt wird, ist sachlich nicht zu beanstanden, trotzdem liegt wohl auch hier eine Interpolation vor. Bereits Seckel ${ }^{1}$ ) hat den "procurator qui ad agendum tantum datus est" für verdächtig erklärt als Pendant zu dem sicher kompilatorischen „procurator tantum ad actionem factus". Setzen wir zunächst

1) Handlexikon S. 464. 
einmal hypothetisch den cognitor ein, so würde auch für diesen unsere Entscheidung passen bis zu den Worten si vero non in rem suam; die Worte dolum praesentem in exceptione conferendum wären für den cognitor falsch, für ihn müßte die Entscheidung lauten: de dolo eius excipi non posse. Eigentümlich nun, daß dieser Satz, der auf den cognitor - diesen wie gesagt hypothetisch eingesetzt nicht passen würde, stilistische Mängel aufweist: 1. in exceptione statt in exceptionem; dies scheint kein Schreibfehler der Abschreiber zu sein, da Florentina wie Vulgaten exceptione lesen. 2. dolum conferre in exceptionem ist eine ungewöhnliche Ausdrucksweise, die in den Pandekten sich allein an dieser Stelle findet. ${ }^{1}$ ) 3 . dolus praesens ist ungenau gesprochen, welche Gegenwart ist gemeint? Wie man sich klassisch ausdrückt, zeigt das vorhin besprochene Fr. 11 (si post litem contestatam dolo quid fecerit); schon die Verfasser der Basiliken haben unser Fragment als zu ungenau redend empfunden. ${ }^{2}$ ) Berücksichtigt man nun außerdem, daß für eine Prozeßvertretung in rem suam sich die Cognitur viel besser eignete ${ }^{3}$ ), daß der procurator in rem suam eben deshalb zwar theoretisch möglich war, praktisch aber gewiß selten genug vorkam, da $\beta$ der procurator in rem suam im Corpus iuris vielfach (wahrscheinlich immer) unecht ist, daß außerhalb des Corpus iuris ein Beleg für den procurator in rem suam, soweit mir bekannt, vollständig fehlt ${ }^{4}$ ), daß endlich das "datus" unseres Textes auf den cognitor, nicht den procurator weist ${ }^{5}$ ), - so erscheint es nicht als gewagt, auch im $\S 18$ den cognitor einzusetzen. Der Satz dolum praesentem - conferendum, dessen stilistische Anstände soeben namhaft gemacht worden sind, ist kompilatorisch, die Kompilatoren mußten diesen Satz schreiben, weil die Entscheidung für den cognitor ganz anders weiterlief.

1) Vocabularium Jur. Rom. 1, 906 Z. 14. - 2) Bas. (51, 4) 4, 18 Heimb. 5,96: sed in litem dati procuratoris solus dolus post litem contestatam commissus nocet. - ${ }^{3}$ ) Eisele, Lognitur und Procuratur S. 70. - ) Eisele S. 71. Gaius 2, 39 zählt kaum, da hier an den procurator in rem suam freilich gedacht ist, aber nur flüchtig und wohl nur als an etwas theoretisch Mögliches. - 5) Le nel, Palingenesie 1, 513 N. 1: 'procuratorem dare' videtur ab usu abhorrere; Rosenberg 308 . 
Nachdem er vom dolus des cognitor gesprochen, wandte sich Ulpian wohl zum procurator und schloß sich hier gewiß. völlig an Neraz an. Die Kompilatoren konnten natürlich diese Ausführungen nicht mehr brauchen, da sie ja gerade schon vom procurator gehandelt hatten; sie setzten daher an die Stelle des einfachen procurator den procurator omnium bonorum. Der Schlußsatz des $\$ 18$ si autem is procurator rel. verrät sich als kompilatorisch durch seine Ungenauigkeit. Entweder nämlich der procurator hat die Forderung, die er jetzt einklagt, selbst durch ein von ihm geschlossenes Geschäft erworben: dann ist die Entscheidung allerdings richtig, es. war aber überflüssig den procurator omnium bonorum herauszuheben, für den Spezialprokurator gilt das Gleiche. Oder aber die Forderung hatte der Prinzipal durch ein eigenes Geschäft erworben, nunmehr ernennt er den A zum procurator omnium bonorum, der jetzt die Forderung einklagt; in diesem Falle ist nicht abzusehen, wieso der dolus des procurator im weiteren Umfange als beim Spezialprokurator hätte geltend gemacht werden können.

Das Ergebnis dieser Ausführungen ist, obwohl die Interpolation dieser Fragmente noch nicht bekannt war, gleichwohl nicht neu ${ }^{1}$ ): weder der dolus des cognitor noch der des procurator schadet dem Vertretenen; nicht der dolus des cognitor, denn ihm gegenüber kann sein dolus nicht geltend gemacht werden (es sei denn er wäre in rem suam bestellt); nicht der dolus des procurator, denn diesem darf zwar sein dolus, den er sich nach der litis contestatio zu schulden kommen ließ, geltend gemacht werden, da aber seine Prozeßführung die actio des Herrn nicht konsumiert, so schadet diese exceptio dem Herrn nicht. Soweit man im späteren klassischen Recht auch dem procurator Konsumptionsmacht verlieh, mußte folgerichtig das Durchgreifen dieser exceptio doli auch ihm gegenüber versagt werden. Belege für diesen Satz fehlen, ausdrücklich das Gegenteil sagt die berühmte Verordnung des Alexander Severus:

$$
\text { C. }(2,12) 10 \text { : }
$$

Si procurator ad unam speciem constitutus officium mandati egressus est, id quod gessit nullum domino prae-

1) So schon Koschaker a.a. 0 . 
iudicium facere potuit. quod si plenam potestatem agendi habuit, rem iudicatam rescindi non oportet, cum, si quid fraude vel dolo egit, convenire eum more iudiciorum non prohiberis.

Hier wird allerdings gesagt: wenn der procurator dolos handelte und seine Klage infolgedessen abgewiesen wurde, so schadet dies dem Vertretenen; dieser hat keine Klage mehr gegen den Dritten und kann sich nur an den procurator halten. Doch die Verordnung ist schwerlich echt: unschöner Stil ist es, das Abstraktum (id quod gessit) als Subjekt zu nehmen, diese Worte würden besser ganz fehlen. Auf den ersten Blick scheint die Stelle Spezial- und Generalprokurator gegenüberzustellen, gemeint ist aber ein ganz anderer Gegensatz: Utherschreitung des Mandats und Verbleiben innerhalb seiner Grenzen ${ }^{1}$ ); si plenam potestatem habuit soll heißen: wenn er Generalvollmacht hatte und infolgedessen in den Grenzen des Mandats blieb, aber die Hauptsache ist den Kompilatoren in der Feder stecken geblieben. „rem iudicatam rescindi non oportet": daß das Urteil vom Prinzipal nicht angefochten werden kann, versteht sich von selbst, die Worte müßten also bedeuten, daß die Klage des Prinzipals gegen den Dritten durch den Prokurator konsumiert worden ist $^{2}$ ); es ist aber klassischen Juristen m. F. nicht zuzutrauen, $\mathrm{da} B$ sie diesen einfachen Gedanken in so grundverkehrter, laienhafter Weise ausgesprochen haben sollten. Die Verordnung verdient keineswegs das Vertrauen, das ihr die Rechtshistoriker bislang entgegengebracht haben. ${ }^{3}$ )

Nicht hierher gehört das folgende von Älteren in diesen Zusammenhang gezogene Fragment:

$$
\text { D. }(44,4) 9 \text { : }
$$

Paulus libro trigensimo secundo ad edictum.

Si procurator cognitor rei pecunia accepta damnari se passus sit et cum domino iudicati agatur, tuebitur se doli mali exceptione. nec hoc, quod acceperit procurator cognitor,

1) Daf dieser Sinn gemeint ist (Eisele 183, Rosenberg 353), glaube ich allerdings auch. - ${ }^{2}$ ) So auch Eisele 184, der aber darin nichts Verdächtiges sieht. - s) Man hält die Verordnung allgemein für echt: Eisele, Rosenberg a.a. O.; Girard, Manuel (5. Aufl.) S. 1028. 
auferri ab eo potest: nam turpiter accepta pecunia iustius penes eum est qui deceptus sit quam qui decepit.

Die Entscheidung redet zwar auch vom dolus des cognitor ${ }^{1}$ ), aber der dolus richtet sich hier nicht wie in den früheren Fällen gegen den Prozeßgegner, sondern gegen den Vertretenen. Der Schlußsatz scheint unecht zu sein, den kolludierenden cognitor als deceptus zu bezeichnen ist ganz unverständlich. Auch stilistisch würde der Klassiker wohl anders geschrieben haben: iustius est turpiter acceptam pecuniam penes eum manere rel. Auffällig ist auch der Moduswechsel (deceptus sit-decepit).

Soviel von der Prozeßvertretung. Wir müssen nunmehr noch einmal zu dem oben ${ }^{2}$ ) wiedergegebenen Fr. 4 zurückkehren, dessen $\$ \S 17$ und 19 noch unerledigt sind.

Der $\S 17$ weist folgende Veränderungen auf: Zunächst handelte der Urtext allein vom Sklaven, nicht auch rom Hauskind. Man sieht dies schon daraus, daß die Entscheidung ausschließlich für den Sklaven gefällt wird, auch ist die Phrase vel alterius - subiectae ganz unmöglich: sie läßt nämlich den Juristen sagen: wir können wegen des dolus der unsrer Gewalt Untergebenen excipieren, was offenbar Unsinn ist. "Wir" können nur wegen des dolus fremder Gewaltuntergebener excipieren. Schon die in solchen Dingen nichts übersehende Glosse hat den Fehler erkannt, ihn freilich zugleich in ganz unhaltbarer Art zu beseitigen gesucht:

Gl. iuri nostro: id est iuri Romanorum. nain de dolo servi mei excipere non possum, sed alius contra me. Item et ego de dolo alterius. Die Entscheidung stammt ferner nicht von Ulpian, da die Worte neque enim esse aequum ein - von den Kompilatoren gestrichenes - $\mathrm{X}$ scribit fordern. Am wichtigsten aber ist natürlich die Veränderung des Inhalts. Wir begegnen hier der uns bereits bekannten. Unterscheidung von Pekuliargeschäften und Nichtpekuliargeschäften, aber die Rechtsfolgen, die an diese Unterscheidung nach dem überlieferten Text geknüpft werden, sind nicht die, die nach unsern obigen Ausführungen die Klassiker damit zu verbinden pflegen: der dolus des Untergebenen soll stets dem Gewalthaber schaden,

1) Lenel, Paling. Paulus Nr.491. $-{ }^{2}$ ) S. 70. 
mag ein Pekuliargeschäft vorliegen oder nicht. Dieser Satz stimmt überein mit den als interpoliert erwiesenen Teilen von Fr. 16 und Fr. 51 ${ }^{1}$ ) und wird dadurch selbst verdächtig. Weitere Anstände kommen hinzu: statt negotium quod geritur müßte es heißen negotium quod gestum est, admissus sit - intervenisset ist unbegründeterTempuswechsel, vor allem aber erregt die schwersten Bedenken der Satz neque enim esse aequum servi dolum amplius domino nocere, quam in quo opera eius esset usus. Als wörtliche Übersetzung dieses Satzes würde sich - da, ,in quo" aufzulösen ist in "in eo negotio in quo" - folgendes ergeben: es ist unbillig, daß der dolus des Sklaven dem Herrn weiter schadet als in dem Geschäft, bei dem der Herr den Sklaven gebraucht hat. Aber davon ist nach dem Vorangehenden hier gar nicht die Rede! Es steht gar nicht zur Erörterung, ob der dolus des Sklaven auch bei der Geltendmachung anderer Ansprüche als der aus dem Sklavengeschäft entsprungenen entgegengehalten werden kann; die Entscheidung setzt ja ausdrücklich voraus, daß aus dem vom Sklaven geschlossenen Geschäfte geklagt wird (ex eorum negotio actio intenditur), nur ob dieser Klage gegenüber der dolus des Sklaven entgegengesetzt werden kann, ist die Frage. In der Tat soll der fragliche Satz auch gar nicht das besagen, was die wörtliche Übersetzung ergibt, er soll sagen: der dolus, den sich der Sklave nicht bei dem ron ihm geschlossenen Nichtpekuliargeschäft hat zu schulden kommen lassen, kann gegenüber der Klage aus diesem Geschäft nicht geltend gemacht werden. Um das zu sagen, hätte aber etwa geschrieben werden müssen: neque enim esse aequum servi dolum domino aliter nocere quam si in negotio eo admissus esset, in quo dominus opera servi esset usus. Den Kompilatoren ist wieder das Wichtigste in der Feder stecken geblieben. Selbst wenn wir aber den Satz so verstehen, wie ihn die Kompilatoren wohl verstanden wissen wollten, so widerspricht er doch der Eingangsentscheidung. Da nämlich nach der Stelle ein "opera servi uti" seitens des Herrn nicht vorliegen soll bei einem Pekuliargeschäft des Sklaven, so müßte bei diesem Geschäft

1) Oben S. 55 und S. 45 . 
der Sklavendolus für den Herrn unschädlich sein: gerade das Gegenteil sagt der Anfang des Paragraphen.

Nach alledem muß auch der $\$ 17$ für interpoliert erklärt werden: Ulpian gab die exceptio doli nur beim Pekuliargeschäft, beim Nichtpekuliargeschäft schadet dem Herrn nur sein eigener dolus. Soviel halte ich für sicher; die Rekonstruktion des Schlusses des Paragraphen ist Hypothese, aber auch ziemlich gleichgültig. Ulpians Gewährsmann begründete vielleicht die Abweisung der exceptio doli beim Nichtpekuliargeschäft damit, es sei unbillig, daß der Sklavendolus dem Herrn schade, wenn der Herr sich beim Abschluß des Geschäftes der Dienste des Sklaven bedient hatte; die Kompilatoren wollten diese Begründung nicht ganz streichen, konnten sie aber auch nicht unverändert lassen.

Interpoliert ist schließlich auch der $\$ 19$. Er handelt nicht von einer Delegation ${ }^{1}$ ), sondern von einem einfachen Mandat; die Entscheidung besagt: wenn der procurator Seius aus der von ihm geschlossenen Stipulation klagt, so kann ihm sowohl sein eigener dolus als der des ersten Mandanten entgegengehalten werden. Damit gerät aber die Entscheidung in Widerspruch mit D. $(21,1) 51,1$, wo ausdrücklich die exceptio aus dem dolus des Mandanten verworfen wird. ${ }^{2}$ ) Die Glosse hat darum die Worte "de meo dolo" auf den Fall beziehen wollen, daß der Mandant mit der an ihn zedierten Klage vorgeht,

Gl. esse: contra Seium et contra me, si cessa actione agam.

und diese Auslegung ist auch von Neueren vertreten worden. ${ }^{3}$ ) Doch diese Ausflucht ist unmöglich. Es ist zu deutlich gesagt, wessen Klage gegenüber der dolus geltend gemacht werden soll: stipulatus a te Seius est et iudicium edidit (also Seius!). Um die Klage des procurator und nur um diese handelt es sich. Zu dem unbehebbaren sachlichen Wider-

1) Dies nahm Mühlenbruch S. 124f. an, um den Widerspruch zu beseitigen: "wenn sich jemand wissend eine Schuld überweisen lief, welcher die exceptio doli entgegenstand, so konnte man - freilich immer nur uneigentlich - sagen, ihm werde diese Einrede aus der Person des Delegierenden entgegengesetzt werden ${ }^{*}$. Dagegen mit Recht Salpius, Novation und Delegation S. 430; Pernice, Labeo 1, 502. $-{ }^{2}$ ) Oben S. 45. - ${ }^{3}$ ) Z. B. Pernice a. a. O.; A. Sch mid 2, 189 N. 54. 
spruch kommen zwei weitere Mängel: 1. warum ist nur vom dolus des ersten, nicht auch des zweiten Mandanten die Rede? Sehr richtig frägt so schon die Glosse.

GI. est: sed numquid et de dolo Titii, si ei actio cedatur? Dicam sic.

2. Sowohl - als auch heißt tam - quam, nicht wie hier tam - quam et. Nach alledem halte ich auch den $\S 19$ für unecht; Labeo wird wie African nur die exceptio aus dem dolus des procurator zugelassen haben, die Kompilatoren aber wollten, wie wir schon früher sahen ${ }^{1}$ ), auch den dolus des Prinzipals schädlich sein lassen. ${ }^{2}$ )

\section{Irrtum beim Kauf.}

D. $(18,1) 12$ :

Pomponius libro trigensimo primo ad Quintum Mucium.

In huiusmodi autem quaestionibus personae ementium et vendentium spectari debent, non eorum quibus adquiritur ex eo contractu actio: nam si servus meus vel filius, qui in mea potestate est, me praesente suo nomine emat, non est quaerendum, quid ego existimem, sed quid ille qui contrahit.

1) Oben S. 54,61. - ${ }^{2}$ ) Der Vollständigkeit halber mag noch kurz behandelt werden D. $(44,4) 5,3$ : Paulus libro septuagensimo ad edictum. Actoris, qui exigendis pecuniis praepositus est, etiam posterior dolus domino nocet. Das Fragment, das bei den Dogmatikern eine gewisse Rolle gespielt hat (Mitteis, Stellvertretung 288 N. 380 und dort Zit.), ist für den Historiker nur von geringem Werte, da die Kompilatoren den Text gar zu abgerissen überliefert haben. Unter dem actor ist natürlich ein Sklave zu verstehen, dem der Herr die Einkassierung der Gelder aufgetragen hat; was aber heist etiam posterior dolus? Sehr richtig bemerkte schon Cuiacius (Obs. 25, 27): quod est obscurum valde; nec enim apparet evidenter, quid hic faciat ille articulus netiam ${ }^{4}$, nec cuius doli comparatione nposteriorem ${ }^{*}$ dolum dicat, neque vero ullus est, qui id nobis liquido explicaverit. C.'s eigene Erklärung ist aber gleichfalls unbefriedigend. Vielleicht gab der Zusammenhang Aufschluf, aus dem die Stelle ja völlig herausgerissen ist (so auch Lenel Paling. Paulus 788-792). Möglich aber auch, daßs die Kompilatoren eingegriffen haben: nach dem interpolierten Teile von Fr. $4 \S 17$ (oben S. 70) schadet beim Pekuliargeschäft der Sklavendolus etiam si postea aliquis dolus intervenisset; an diese Phrase klingt unser posterior dolus an. Genaues läßt sich bei einem so kurzen Schnitzel nicht ermitteln, das Fragment bleibt am besten ganz beiseite. 
Die Entscheidung, die wohl nicht nur in der Kompilation vom Irrtume handelte ${ }^{1}$ ), entspricht in der Hauptsache den klassischen Prinzipien: wenn der Untergebene suo nomine handelte (für den Klassiker muß das dasselbe sein, wie peculiari nomine), so wird allein seine Person berücksichtigt. Der Eingangssatz redet. freilich allgemeiner; möglich, daß die Kompilatoren die Beschränkung auf das Pekuliargeschäft gestrichen haben, möglich aber auch, daß Pomponius hinter contrahit fortfuhr: sane si meo nomine emat... Utber das klassische Recht kann kein Zweifel sein.

\section{Irrtum beim Besitzerwerb.}

D. $(41,2) 34,1$ u. 2 :

Ulpianus libro septimo disputationum.

Sed si non mihi, sed procuratori meo possessionem tradas, videndum est, si ego errem, procurator meus non erret, an mihi possessio adquiratur. et cum placeat ignoranti andquiri, poterit et erranti placet non adquiri possessionem. Sed si procurator meus erret, ego non errem, magis est ut adquiram possessionem.

2. Servus quoque meus ignoranti mihi adquiret possessionem.

Auch diese Stelle ist eine alte crux. ${ }^{2}$ ) Von den beiden im $\$ 1$ berichteten Entscheidungen ist aber nach dem bisherigen nicht etwa die zweite, sondern die erste auffällig: man möchte erwarten, daß die Person des Prokurator, der hier direkter Vertreter ist, ganz unberücksichtigt bleibt. M. E. sind auch gegen diese erste Entscheidung Verdachtsgründe gegeben.

1. Statt sed müßte es sed et heißen; natürlich kann das an sich ein Schreibfehler sein, bemerkenswert ist immerhin, daß auch in den Vulgaten das et fehlt. Hält man sich den sachlich bedenklichen, zum mindesten schwierigen Inhalt der Stelle gegenwärtig, so erregt dieser stilistische Mangel den

1) Ohne Grund bezweifelt das Herrmann, Zeitschr. f. Zivilrecht und Prozer N. F. 7, 270. - ${ }^{2}$ ) Windscheid, Pand. 1 \$ 73 N.21. Windscheids eigene Auslegung ist historisch ganz unmöglich. 
Verdacht, daß die Entscheidung vor sed im Urtext wahrscheinlich den Besitzerwerb leugnete, worauf dann das sed ganz berechtigt war.

2. Der entscheidende Satz: et cum placeat ignoranti adquiri, poterit et erranti ist in dem abgerissenen Stil der Interpolationen geschrieben, trotz des Kasuswechsels fehlt beidemal das Subjekt.

3. Die Logik dieses Satzes ist anfechtbar. Wenn die herrschende Lehre Recht hat - und sie scheint mir in der Tat in den Quellen begründet - daß auch zum Besitzerwerb durch den Prokurator entweder der Auftrag oder die nachträgliche Genehmigung des Vertretenen erforderlich ist, so hat der Satz ignoranti possessio adquiritur nur den Sinn, daß beim Vorliegen eines mehr oder minder bestimmten Mandats die Kenntnis des Vertretenen von der Vollziehung des Auftrags zum Besitzerwerb nicht erforderlich ist ${ }^{1}$ ); keineswegs aber hat der Satz den Sinn, daß auf den Willen des Vertretenen überhaupt nichts ankommt. Diesen letzteren (abgelehnten) Sinn müßte aber der Satz offensichtlich haben, wenn aus ihm etwas für die Unbeachtlichkeit des Irrtums des Vertretenen gefolgert werden sollte.

4. Im $\S 2$ heißt es: a uch der Sklave erwirbt dem Herrn ohne sein Wissen. Natürlich zielt das "quoque" auf einen vorangehenden Satz procurator meus ignoranti mihi adquiret possessionem, nach diesem Satz sucht man aber vergebens. Um für das quoque Anschluß zu gewinnen, muß man in den begründenden Nebensatz einer ganz anderen Entscheidung im vorletzten Satze zurückspringen. Solcher Stil ist Ulpian schwerlich zuzutrauen. Wahrscheinlich hat vor $\$ 2$ der desiderierte Satz wirklich gestanden; er gab den Kompilatoren Anlaß, die erste Entscheidung des $\S 1$ zu ändern, und nachdem sie ihn hier als Begründung verwendet hatten, glaubten sie ihn im Folgenden streichen zu können. Jedenfalls glaube ich, daß auch diese Stelle das Vertrauen, das man bisher ihrer Echtheit entgegengebracht hat, wenig verdient.

1) So Mitteis, Röm. Privatrecht 1,213; Bruns, Besitzerwerb durch Stellvertreter 19, vg], auch Windscheid $1 \S 155$ N. 9. 
80 Fritz Schulz, Scientia, Dolus u. Error bei der Stell vertretung usw.

Unser Quellenmaterial ist damit, soweit ich sehe, erschöpft, D. $\left.(50,17) 74^{1}\right)$ und $\left.(22,6) 5^{2}\right)$, die früher vielfach bei der Behandlung unserer Frage angezogen wurden, reden nicht von der Stellvertretung.

Die im Eingange aufgestellten Thesen dürften damit so wahrscheinlich gemacht sein, als es eben möglich ist, wenn die Quellenbasis so schmal wie hier, und die Uberlieferung außerhalb des Corpus iuris nirgends einen Stützpunkt gibt. Auch wer aber gegenüber meinen Nachweisen über das klassische Recht skeptisch bleibt, wird wenigstens eins anerkennen müssen: die Tatsache, daß die Kompilatoren hier eingegriffen haben und auf sie daher die Widersprüche unter den Quellenentscheidungen zurückzuführen sind. Schon diese Erkenntnis bedeutet gegenüber der bisherigen Literatur einen Fortschritt: der Kreis der dogmatischen Vereinigungsversuche ist jetzt jedenfalls für immer geschlossen.

1) Vgl. Lene 1, Paling. Papinian 66. $-{ }^{2}$ ) Lene], Paling. Terentius 1 ; Curtius 92. 